\title{
FRACTAL MODELS FOR NORMAL SUBGROUPS OF SCHOTTKY GROUPS
}

\author{
JOHANNES JAERISCH
}

\begin{abstract}
For a normal subgroup $N$ of the free group $\mathbb{F}_{d}$ with at least two generators, we introduce the radial limit set $\Lambda_{r}(N, \Phi)$ of $N$ with respect to a graph directed Markov system $\Phi$ associated to $\mathbb{F}_{d}$. These sets are shown to provide fractal models of radial limit sets of normal subgroups of Kleinian groups of Schottky type. Our main result states that if $\Phi$ is symmetric and linear, then we have that $\operatorname{dim}_{H}\left(\Lambda_{r}(N, \Phi)\right)=\operatorname{dim}_{H}\left(\Lambda_{r}\left(\mathbb{F}_{d}, \Phi\right)\right)$ if and only if the quotient group $\mathbb{F}_{d} / N$ is amenable, where $\operatorname{dim}_{H}$ denotes the Hausdorff dimension. This extends a result of Brooks for normal subgroups of Kleinian groups to a large class of fractal sets. Moreover, we show that if $\mathbb{F}_{d} / N$ is nonamenable, then $\operatorname{dim}_{H}\left(\Lambda_{r}(N, \Phi)\right)>\operatorname{dim}_{H}\left(\Lambda_{r}\left(\mathbb{F}_{d}, \Phi\right)\right) / 2$, which extends results by Falk and Stratmann and by Roblin.
\end{abstract}

\section{Introduction AND StATEMEnt of RESUlts}

In this paper we introduce and investigate linear models for the Poincaré series and the radial limit set of normal subgroups of Kleinian groups of Schottky type. Here, a linear model means a linear graph directed Markov system (GDMS) associated to the free group $\mathbb{F}_{d}=\left\langle g_{1}, \ldots, g_{d}\right\rangle$ on $d \geq 2$ generators. Precise definitions are given in Section 2.2. but briefly, such a system $\Phi$ is given by the vertex set $V:=\left\{g_{1}, g_{1}^{-1}, \ldots, g_{d}, g_{d}^{-1}\right\}$, edge set $E:=\left\{(v, w) \in V^{2}: v \neq w^{-1}\right\}$ and by a family of contracting similarities $\left\{\phi_{(v, w)}:(v, w) \in E\right\}$ of the Euclidean space $\mathbb{R}^{d}$, for $d \geq 1$, such that for each $(v, w) \in E$ the contraction ratio of the similarity $\phi_{(v, w)}$ is independent of $w$. We denote this ratio by $c_{\Phi}(v)$. Also, we say that $\Phi$ is symmetric if $c_{\Phi}(g)=c_{\Phi}\left(g^{-1}\right)$ for all $g \in V$. In order to state our first two main results, we must also make two further definitions. For this, we extend $c_{\Phi}$ to a function $c_{\Phi}: \mathbb{F}_{d} \rightarrow \mathbb{R}$ by setting $c_{\Phi}(g):=\prod_{i=1}^{n} c_{\Phi}\left(v_{i}\right)$, where $n \in \mathbb{N}$ and $\left(v_{1}, \ldots, v_{n}\right) \in V^{n}$ refers to the unique representation of $g$ as a reduced word. Also, for each subgroup $H$ of $\mathbb{F}_{d}$, we introduce the Poincaré series of $H$ and the exponent of convergence of $H$ with respect to $\Phi$ which are defined for $s \geq 0$ by

$$
P(H, \Phi, s):=\sum_{h \in H}\left(c_{\Phi}(h)\right)^{s} \quad \text { and } \quad \delta(H, \Phi):=\inf \{t \geq 0: P(H, \Phi, t)<\infty\} .
$$

Our first main result gives a relation between amenability and the exponent of convergence.

Received by the editors December 15, 2012.

2010 Mathematics Subject Classification. Primary 37C45, 30F40; Secondary 37C85, 43A07.

Key words and phrases. Normal subgroups of Kleinian groups, exponent of convergence, graph directed Markov system, amenability, Perron-Frobenius operator, random walks on groups.

The author was supported by the research fellowship JA 2145/1-1 of the German Research Foundation (DFG). 
Theorem 1.1. Let $\Phi$ be a symmetric linear GDMS associated to $\mathbb{F}_{d}$. For every normal subgroup $N$ of $\mathbb{F}_{d}$, we have that

$$
\delta\left(\mathbb{F}_{d}, \Phi\right)=\delta(N, \Phi) \text { if and only if } \mathbb{F}_{d} / N \text { is amenable. }
$$

Our second main result gives a lower bound for the exponent of convergence $\delta(N, \Phi)$.

Theorem 1.2. Let $\Phi$ be a symmetric linear GDMS associated to $\mathbb{F}_{d}$. For every non-trivial normal subgroup $N$ of $\mathbb{F}_{d}$, we have that

$$
\delta(N, \Phi)>\delta\left(\mathbb{F}_{d}, \Phi\right) / 2 .
$$

Our next results study certain limit sets which provide fractal models of radial limit sets of Kleinian groups. More precisely, for a GDMS $\Phi$ associated to $\mathbb{F}_{d}$ and a subgroup $H$ of $\mathbb{F}_{d}$, we will consider the radial limit set $\Lambda_{\mathrm{r}}(H, \Phi)$ of $H$ and the uniformly radial limit set $\Lambda_{\mathrm{ur}}(H, \Phi)$ of $H$ with respect to $\Phi$ (see Definition 2.10).

Proposition 1.3. Let $\Phi$ be a linear $G D M S$ associated to $\mathbb{F}_{d}$. For every normal subgroup $N$ of $\mathbb{F}_{d}$, we have that

$$
\delta(N, \Phi)=\operatorname{dim}_{H}\left(\Lambda_{\mathrm{ur}}(N, \Phi)\right)=\operatorname{dim}_{H}\left(\Lambda_{\mathrm{r}}(N, \Phi)\right) .
$$

The following corollary is an immediate consequence of Theorem 1.1, Theorem 1.2 and Proposition 1.3.

Corollary 1.4. Let $\Phi$ be a symmetric linear $G D M S$ associated to $\mathbb{F}_{d}$. For every normal subgroup $N$ of $\mathbb{F}_{d}$, we have that

$$
\operatorname{dim}_{H}\left(\Lambda_{\mathrm{r}}(N, \Phi)\right)=\operatorname{dim}_{H}\left(\Lambda_{\mathrm{r}}\left(\mathbb{F}_{d}, \Phi\right)\right) \text { if and only if } \mathbb{F}_{d} / N \text { is amenable. }
$$

Moreover, if $N$ is non-trivial, then we have that

$$
\operatorname{dim}_{H}\left(\Lambda_{\mathrm{r}}(N, \Phi)\right)>\operatorname{dim}_{H}\left(\Lambda_{\mathrm{r}}\left(\mathbb{F}_{d}, \Phi\right)\right) / 2 .
$$

Let us now briefly summarize the corresponding results for normal subgroups of Kleinian groups, which served as the motivation for our main results in this paper. A more detailed discussion of Kleinian groups and how these relate to the concept of a GDMS will be given in Section 5. We start by giving a short introduction to Kleinian groups.

Recall that, for $m \in \mathbb{N}$, an $(m+1)$-dimensional hyperbolic manifold can be described by the hyperbolic $(m+1)$-space $\mathbb{D}^{m+1}:=\left\{z \in \mathbb{R}^{m+1}:|z|<1\right\}$ equipped with the hyperbolic metric $d$ and quotiented by the action of a Kleinian group $G$. The Poincaré series of $G$ and the exponent of convergence of $G$ are for $s \geq 0$ given by

$$
P(G, s):=\sum_{g \in G} \mathrm{e}^{-s d(0, g(0))} \quad \text { and } \quad \delta(G):=\inf \{t \geq 0: P(G, t)<\infty\} .
$$

A normal subgroup $N$ of a Kleinian group $G$ gives rise to an intermediate covering of the associated hyperbolic manifold $\mathbb{D}^{m+1} / G$. It was shown by Brooks in Bro85] that if $N$ is a normal subgroup of a convex cocompact Kleinian group $G$ such that $\delta(G)>m / 2$, then we have that

$$
\delta(N)=\delta(G) \text { if and only if } G / N \text { is amenable. }
$$

Moreover, Falk and Stratmann FS04 showed that for every non-trivial normal subgroup $N$ of a non-elementary Kleinian group $G$ we have $\delta(N) \geq \delta(G) / 2$. Using 
different methods, Roblin ([Rob05]) proved that if $G$ is of $\delta(G)$-divergence type, that is, if $P(G, \delta(G))=\infty$, then we have

$$
\delta(N)>\delta(G) / 2 .
$$

Another proof of (1.2) can be found in BTMT12] for a convex cocompact Kleinian group $G$, where it was also shown that $\delta(N)$ can be arbitrarily close to $\delta(G) / 2$.

Note that our results stated in Theorem 1.1 and Theorem 1.2 extend the assertions given in (1.1) and (1.2) for Kleinian groups.

Remark. Note that in Theorem 1.1 there is no restriction on $\delta\left(\mathbb{F}_{d}, \Phi\right)$, whereas for the proof of (1.1) it was vital to assume that $\delta(G)>m / 2$. It was conjectured by Stratmann Str06 that this assumption can be removed from Brooks' Theorem. In fact, it was shown by Sharp in [Sha07. Theorem 2] that if $G$ is a finitely generated Fuchsian group, that is, for $m=1$, and if $N$ is a normal subgroup of $G$, then amenability of $G / N$ implies $\delta(G)=\delta(N)$. Recently, Stadlbauer Sta13] showed that the equivalence in (1.1) extends to the class of essentially free Kleinian groups with arbitrary exponent of convergence $\delta(G)$.

Finally, let us turn our attention to limit sets of Kleinian groups. For a Kleinian group $G$, the radial limit set $L_{\mathrm{r}}(G)$ and the uniformly radial limit set $L_{\mathrm{ur}}(G)$ (see Definition 5.1) are both subsets of the boundary $\mathbb{S}:=\left\{z \in \mathbb{R}^{m+1}:|z|=1\right\}$ of $\mathbb{D}^{m+1}$. By a theorem of Bishop and Jones ([BJ97, Theorem 1.1]; cf. Str04]), we have for every Kleinian group $G$ that

$$
\delta(G)=\operatorname{dim}_{H}\left(L_{\mathrm{ur}}(G)\right)=\operatorname{dim}_{H}\left(L_{\mathrm{r}}(G)\right),
$$

where $\operatorname{dim}_{H}$ denotes the Hausdorff dimension with respect to the Euclidean metric on $\mathbb{S}$. Combining (1.1) and (1.3) then shows that for every normal subgroup $N$ of a convex cocompact Kleinian group $G$ for which $\delta(G)>m / 2$, we have

$$
\operatorname{dim}_{H}\left(L_{\mathrm{r}}(N)\right)=\operatorname{dim}_{H}\left(L_{\mathrm{r}}(G)\right) \text { if and only if } G / N \text { is amenable. }
$$

We would like to point out that there is a close analogy between the results on radial limit sets of Kleinian groups stated in (1.3) and (1.4), and our results in the context of linear GDMSs associated to free groups stated in Proposition 1.3 and Corollary 1.4 .

Let us now further clarify the relation between GDMSs associated to free groups and Kleinian groups of Schottky type (see Definition [5.2). For this, recall that a Kleinian group of Schottky type $G=\left\langle g_{1}, \ldots, g_{d}\right\rangle$ is isomorphic to a free group. In Definition 5.3 we introduce a canonical GDMS $\Phi_{G}$ associated to $G$. We will then show in Proposition 5.6 that for every non-trivial normal subgroup $N$ of $G$ we have that

$$
L_{\mathrm{r}}(N)=\Lambda_{\mathrm{r}}\left(N, \Phi_{G}\right) \quad \text { and } \quad L_{\mathrm{ur}}(N)=\Lambda_{\mathrm{ur}}\left(N, \Phi_{G}\right) .
$$

This shows that our fractal models of radial limit sets of Kleinian groups of Schottky type can be thought of as a replacement of the conformal generators of the Kleinian group by similarity maps. Our main results show that several important properties of Kleinian groups extend to these fractal models.

Let us now end this introductory section by briefly summarizing the methods used to obtain our results and how this paper is organized. Theorem 1.1 and Theorem 1.2 are based on and extend results of Woess Woe00 and Ortner and Woess OW07, which in turn refer back to work of Pólya Pól21 and Kesten Kes59b, Kes59a]. Specifically, we provide generalizations of OW07] for weighted 
graphs. Our new thermodynamic formalism for group-extended Markov systems (see Section 3) characterizes amenability of discrete groups in terms of topological pressure and the spectral radius of the Perron-Frobenius operator acting on a certain $L^{2}$-space.

The paper is organized as follows. In Section 2 we collect the necessary background on thermodynamic formalism, GDMSs and random walks on graphs. In Section 3 , we prove a thermodynamic formalism for group-extended Markov systems, which is also of independent interest. Using the results of Section 3 we prove our main results in Section 4 . Finally, in Section 5 we provide the background on Kleinian groups of Schottky type, which has motivated our results.

After having finished this paper, Stadlbauer (Sta13] ) proved a partial extension of Theorem 3.21 (see Remark 3.23). Moreover, in Jae13 the author has extended Lemma 4.1 and Theorem 1.2 in order to give a short new proof of (1.2) for Kleinian groups. Recently, the author ([Jae14a], Jae14b] ) has further generalized Theorem 3.21 and Corollary 3.22. Also, a larger class of conformal fractals has been investigated in Jae14c.

\section{Preliminaries}

2.1. Symbolic thermodynamic formalism. Throughout, the underlying symbolic space for the symbolic thermodynamic formalism will be a Markov shift $\Sigma$, which is given by

$$
\Sigma:=\left\{\omega:=\left(\omega_{1}, \omega_{2}, \ldots\right) \in I^{\mathbb{N}}: a\left(\omega_{i}, \omega_{i+1}\right)=1 \text { for all } i \in \mathbb{N}\right\},
$$

where $I$ denotes a finite or countable infinite alphabet, the matrix $A=(a(i, j)) \in$ $\{0,1\}^{I \times I}$ is the incidence matrix and the shift map $\sigma: \Sigma \rightarrow \Sigma$ is defined by $\sigma\left(\left(\omega_{1}, \omega_{2}, \ldots\right)\right):=\left(\omega_{2}, \omega_{3}, \ldots\right)$, for each $\left(\omega_{1}, \omega_{2}, \ldots\right) \in \Sigma$. We always assume that for each $i \in I$ there exists $j \in I$ such that $a(i, j)=1$. The set of $A$-admissible words of length $n \in \mathbb{N}$ is given by

$$
\Sigma^{n}:=\left\{\left(\omega_{1}, \ldots, \omega_{n}\right) \in I^{n}: a\left(\omega_{i}, \omega_{i+1}\right)=1 \text { for all } i \in\{1, \ldots, n-1\}\right\},
$$

and we set $\Sigma^{0}:=\{\varnothing\}$, where $\varnothing$ denotes the empty word. Note that $\varnothing$ will also be used to denote the empty set. The set of all finite $A$-admissible words is denoted by

$$
\Sigma^{*}:=\bigcup_{n \in \mathbb{N}} \Sigma^{n}
$$

Let us also define the word length function $|\cdot|: \Sigma^{*} \cup \Sigma \cup\{\varnothing\} \rightarrow \mathbb{N}_{0} \cup\{\infty\}$, where for $\omega \in \Sigma^{*}$ we set $|\omega|$ to be the unique $n \in \mathbb{N}$ such that $\omega \in \Sigma^{n}$, for $\omega \in \Sigma$ we set $|\omega|:=\infty$ and $\varnothing$ is the unique word of length zero. For each $\omega \in \Sigma^{*} \cup \Sigma\{\varnothing\}$ and $n \in \mathbb{N}_{0}$ with $n \leq|\omega|$, we define $\omega_{\mid n}:=\left(\omega_{1}, \ldots, \omega_{n}\right)$. For $\omega, \tau \in \Sigma$, we set $\omega \wedge \tau$ to be the longest common initial block of $\omega$ and $\tau$, that is, $\omega \wedge \tau:=\omega_{\mid l}$, where $l:=\sup \left\{n \in \mathbb{N}_{0}: \omega_{\mid n}=\tau_{\mid n}\right\}$. For $\omega \in \Sigma^{n}, n \in \mathbb{N}_{0}$, the cylinder set $[\omega]$ defined by $\omega$ is given by $[\omega]:=\left\{\tau \in \Sigma: \tau_{\mid n}=\omega\right\}$. Note that $[\varnothing]=\Sigma$.

If $\Sigma$ is the Markov shift with alphabet $I$ whose incidence matrix consists entirely of $1 \mathrm{~s}$, then we have that $\Sigma=I^{\mathbb{N}}$ and $\Sigma^{n}=I^{n}$, for all $n \in \mathbb{N}$. Then we set $I^{*}:=\Sigma^{*}$ and $I^{0}:=\{\varnothing\}$. For $\omega, \tau \in I^{*} \cup\{\varnothing\}$, let $\omega \tau \in I^{*} \cup\{\varnothing\}$ denote the concatenation of $\omega$ and $\tau$, which is defined by $\omega \tau:=\left(\omega_{1}, \ldots, \omega_{|\omega|}, \tau_{1}, \ldots, \tau_{|\tau|}\right)$, for $\omega, \tau \in I^{*}$, and if $\omega \in I^{*} \cup\{\varnothing\}$, then we define $\omega \varnothing:=\varnothing \omega:=\omega$. Note that $I^{*}$ is the free semigroup over the set $I$ which satisfies the following universal property: For each semigroup 
$S$ and for every map $u: I \rightarrow S$, there exists a unique semigroup homomorphism $\widehat{u}: I^{*} \rightarrow S$ such that $\widehat{u}(i)=u(i)$, for all $i \in I$ (see [Ber98, Section 3.10]).

Moreover, we equip $I^{\mathbb{N}}$ with the product topology of the discrete topology on $I$, and the Markov shift $\Sigma \subset I^{\mathbb{N}}$ is equipped with the subspace topology. The latter topology on $\Sigma$ is the weakest topology on $\Sigma$ such that for each $j \in \mathbb{N}$ the canonical projection on the $j$-th coordinate $p_{j}: \Sigma \rightarrow I$ is continuous. A countable basis for this topology on $\Sigma$ is given by the cylinder sets $\left\{[\omega]: \omega \in \Sigma^{*}\right\}$. We will use the following metric generating the topology on $\Sigma$. For $\alpha>0$ fixed, we define the metric $d_{\alpha}$ on $\Sigma$ given by

$$
d_{\alpha}(\omega, \tau):=\mathrm{e}^{-\alpha|\omega \wedge \tau|}, \text { for all } \omega, \tau \in \Sigma .
$$

For a function $f: \Sigma \rightarrow \mathbb{R}$ and $n \in \mathbb{N}_{0}$, we use the notation $S_{n} f: \Sigma \rightarrow \mathbb{R}$ to denote the ergodic sum of $f$ with respect to the left-shift map $\sigma$; in other words, $S_{n} f:=\sum_{i=0}^{n-1} f \circ \sigma^{i}$.

Furthermore, the following function spaces will be crucial throughout.

Definition 2.1. We say that a function $f: \Sigma \rightarrow \mathbb{R}$ is bounded if $\|f\|_{\infty}:=$ $\sup _{\omega \in \Sigma}|f(\omega)|$ is finite. We denote by $C_{b}(\Sigma)$ the real vector space of bounded continuous functions on $\Sigma$. We say that $f: \Sigma \rightarrow \mathbb{R}$ is $\alpha$-Hölder continuous, for some $\alpha>0$, if

$$
V_{\alpha}(f):=\sup _{n \geq 1}\left\{V_{\alpha, n}(f)\right\}<\infty,
$$

where for each $n \in \mathbb{N}$ we let

$$
V_{\alpha, n}(f):=\sup \left\{\mathrm{e}^{-\alpha} \frac{|f(\omega)-f(\tau)|}{d_{\alpha}(\omega, \tau)}: \omega, \tau \in \Sigma,|\omega \wedge \tau| \geq n\right\} .
$$

The function $f$ is called Hölder continuous if there exists $\alpha>0$ such that $f$ is $\alpha$-Hölder continuous.

For $\alpha>0$ we also introduce the real vector space

$$
H_{\alpha}(\Sigma):=\left\{f \in C_{b}(\Sigma): f \text { is } \alpha-\text { Hölder continuous }\right\},
$$

which we assume to be equipped with the norm $\|\cdot\|_{\alpha}$ which is given by

$$
\|f\|_{\alpha}:=\|f\|_{\infty}+V_{\alpha}(f) .
$$

We need the following notion of pressure, which was originally introduced in [JKL14, Definition 1.1].

Definition 2.2. For $\varphi, \psi: \Sigma \rightarrow \mathbb{R}$ with $\psi \geq 0, \mathcal{C} \subset \Sigma^{*}$ and $\eta>0$, the $\psi$-induced pressure of $\varphi$ (with respect to $\mathcal{C}$ ) is given by

$$
\mathcal{P}_{\psi}(\varphi, \mathcal{C}):=\limsup _{T \rightarrow \infty} \frac{1}{T} \log \sum_{\omega \in \mathcal{C}: T-\eta<S_{\omega} \psi \leq T} \exp S_{\omega} \varphi,
$$

where we have set $S_{\omega} \varphi:=\sup _{\tau \in[\omega]} S_{|\omega|} \varphi(\tau)$. Note that $\mathcal{P}_{\psi}(\varphi, \mathcal{C})$ is an element of $\overline{\mathbb{R}}:=\mathbb{R} \cup\{-\infty,+\infty\}$.

Remark. It was shown in [JKL14, Theorem 2.4] that the definition of $\mathcal{P}_{\psi}(\varphi, \mathcal{C})$ is in fact independent of the choice of $\eta>0$. For this reason, we do not refer to $\eta>0$ in the definition of the induced pressure.

Notation. If $\psi$ and/or $\mathcal{C}$ is left out in the notation of induced pressure, then we tacitly assume that $\psi=1$ and/or $\mathcal{C}=\Sigma^{*}$, that is, we let $\mathcal{P}(\varphi):=\mathcal{P}_{1}\left(\varphi, \Sigma^{*}\right)$. 
The following fact is taken from [JKL14, Remark 2.11, Remark 2.7].

Fact 2.3. Let $\Sigma$ be a Markov shift over a finite alphabet. If $\varphi, \psi: \Sigma \rightarrow \mathbb{R}$ are two functions such that $\psi \geq c>0$, for some $c>0$, and if $\mathcal{C} \subset \Sigma^{*}$, then $\mathcal{P}_{\psi}(\varphi, \mathcal{C})$ is equal to the unique real number $s \in \mathbb{R}$ for which $\mathcal{P}(\varphi-s \psi, \mathcal{C})=0$. Moreover, we have that

$$
\mathcal{P}_{\psi}(\varphi, \mathcal{C})=\inf \left\{s \in \mathbb{R}: \sum_{\omega \in \mathcal{C}} \mathrm{e}^{S_{\omega}(\varphi-s \psi)}<\infty\right\} .
$$

The next definition goes back to the work of Ruelle and Bowen ([Rue69, Bow75]).

Definition 2.4. Let $\varphi: \Sigma \rightarrow \mathbb{R}$ be continuous. We say that a Borel probability measure $\mu$ is a Gibbs measure for $\varphi$ if there exists a constant $C>0$ such that

$$
C^{-1} \leq \frac{\mu[\omega]}{\mathrm{e}^{S_{|\omega|} \varphi(\tau)-|\omega| \mathcal{P}(\varphi)}} \leq C, \text { for all } \omega \in \Sigma^{*} \text { and } \tau \in[\omega] .
$$

The Perron-Frobenius operator, which we are going to define now, provides a useful tool for guaranteeing the existence of Gibbs measures and for deriving some of the stochastic properties of these measures (see [Rue69, Bow75]).

Definition 2.5. Let $\Sigma$ be a Markov shift over a finite alphabet and let $\varphi: \Sigma \rightarrow \mathbb{R}$ be continuous. The Perron-Frobenius operator associated to $\varphi$ is the operator $\mathcal{L}_{\varphi}$ : $C_{b}(\Sigma) \rightarrow C_{b}(\Sigma)$ which is given, for each $f \in C_{b}(\Sigma)$ and $x \in \Sigma$, by

$$
\mathcal{L}_{\varphi}(f)(x):=\sum_{y \in \sigma^{-1}\{x\}} \mathrm{e}^{\varphi(y)} f(y) .
$$

The following theorem summarizes some of the main results of the thermodynamic formalism for a Markov shift $\Sigma$ with a finite alphabet $I$ (see for instance WWal82] and [MU03, Section 2]). Here, $\Sigma$ is called irreducible if for all $i, j \in I$ there exists $\omega \in \Sigma^{*} \cup\{\varnothing\}$ such that $i \omega j \in \Sigma^{*}$. Moreover, for $k \in \mathbb{N}_{0}$, the $\sigma$-algebra generated by $\left\{[\omega]: \omega \in \Sigma^{k}\right\}$ is denoted by $\mathcal{C}(k)$, and we say that $f: \Sigma \rightarrow \mathbb{R}$ is $\mathcal{C}(k)$-measurable if $f^{-1}(A) \in \mathcal{C}(k)$ for every $A \in \mathcal{B}(\mathbb{R})$, where $\mathcal{B}(\mathbb{R})$ denotes the Borel $\sigma$-algebra on $\mathbb{R}$.

Theorem 2.6. Let $\Sigma$ be an irreducible Markov shift over a finite alphabet and let $\varphi: \Sigma \rightarrow \mathbb{R}$ be $\alpha$-Hölder continuous, for some $\alpha>0$. Then there exists a unique Borel probability measure $\mu$ supported on $\Sigma$ such that $\int \mathcal{L}_{\varphi}(f) d \mu=\mathrm{e}^{\mathcal{P}(\varphi)} \int f d \mu$, for all $f \in C_{b}(\Sigma)$. Furthermore, $\mu$ is a Gibbs measure for $\varphi$ and there exists a unique $\alpha$-Hölder continuous function $h: \Sigma \rightarrow \mathbb{R}^{+}$such that $\int h d \mu=1$ and $\mathcal{L}_{\varphi}(h)=\mathrm{e}^{\mathcal{P}(\varphi)} h$. The measure $h d \mu$ is the unique $\sigma$-invariant Gibbs measure for $\varphi$ and will be denoted by $\mu_{\varphi}$. If $\varphi: \Sigma \rightarrow \mathbb{R}$ is $\mathcal{C}(k)$-measurable, for some $k \in \mathbb{N}_{0}$, then $h$ is $\mathcal{C}(\max \{k-1,1\})$-measurable.

2.2. Graph directed Markov systems. In this section we will first recall the definition of a graph directed Markov system (GDMS), which was introduced by Mauldin and Urbański MU03. Subsequently, we will introduce the notion of a linear GDMS associated to a free group and certain radial limit sets.

Definition 2.7 (Graph directed Markov system). A graph directed Markov system $(G D M S) \Phi:=\left(V,\left(X_{v}\right)_{v \in V}, E, i, t,\left(\phi_{e}\right)_{e \in E}, A\right)$ consists of a finite vertex set $V$, a family of non-empty compact metric spaces $\left(X_{v}\right)_{v \in V}$, a countable edge set $E$, the maps $i, t: E \rightarrow V$ defining the initial and terminal vertex of an edge, a family of 
injective contractions $\phi_{e}: X_{t(e)} \rightarrow X_{i(e)}$ with Lipschitz constants bounded by some $0<s<1$, and an edge incidence matrix $A=(a(e, f)) \in\{0,1\}^{E \times E}$ such that $a(e, f)=1$ implies $t(e)=i(f)$, for all $e, f \in E$. For a GDMS $\Phi$ there exists a canonical coding map $\pi_{\Phi}: \Sigma_{\Phi} \rightarrow \bigoplus_{v \in V} X_{v}$, which is defined by

$$
\bigcap_{n \in \mathbb{N}} \phi_{\omega_{\mid n}}\left(X_{t\left(\omega_{n}\right)}\right)=\left\{\pi_{\Phi}(\omega)\right\}
$$

where $\bigoplus_{v \in V} X_{v}$ denotes the disjoint union of the sets $X_{v}, \phi_{\left.\omega\right|_{n}}:=\phi_{\omega_{1}} \circ \cdots \circ \phi_{\omega_{n}}$ and $\Sigma_{\Phi}$ denotes the Markov shift with alphabet $E$ and incidence matrix $A$. We set

$$
J(\Phi):=\pi_{\Phi}\left(\Sigma_{\Phi}\right), \quad J^{*}(\Phi):=\bigcup_{F \subset E, \operatorname{card}(F)<\infty} \pi_{\Phi}\left(\Sigma_{\Phi} \cap F^{\mathbb{N}}\right),
$$

and refer to $J(\Phi)$ as the limit set of $\Phi$.

The following notion was introduced in [MU03, Section 4].

Definition 2.8. The GDMS $\Phi=\left(V,\left(X_{v}\right)_{v \in V}, E, i, t,\left(\phi_{e}\right)_{e \in E}, A\right)$ is called conformal if the following conditions are satisfied:

(a) For $v \in V$, the phase space $X_{v}$ is a compact connected subset of a Euclidean space $\left(\mathbb{R}^{D},\|\cdot\|\right)$, for some $D \geq 1$, such that $X_{v}$ is equal to the closure of its interior, that is, $X_{v}=\overline{\operatorname{Int}\left(X_{v}\right)}$.

(b) (Open set condition (OSC)) For all $a, b \in E$ with $a \neq b$, we have that

$$
\phi_{a}\left(\operatorname{Int}\left(X_{t(a)}\right)\right) \cap \phi_{b}\left(\operatorname{Int}\left(X_{t(b)}\right)\right)=\varnothing .
$$

(c) For each vertex $v \in V$ there exists an open connected set $W_{v} \supset X_{v}$ such that the map $\phi_{e}$ extends to a $C^{1}$ conformal diffeomorphism of $W_{v}$ into $W_{i(e)}$, for every $e \in E$ with $t(e)=v$.

(d) (Cone property) There exist $l>0$ and $0<\gamma<\pi / 2$ such that for each $x \in X \subset \mathbb{R}^{D}$ there exists an open cone $\operatorname{Con}(x, \gamma, l) \subset \operatorname{Int}(X)$ with vertex $x$, central angle of measure $\gamma$ and altitude $l$.

(e) There are two constants $L \geq 1$ and $\alpha>0$ such that for each $e \in E$ and $x, y \in X_{t(e)}$ we have

$$
|| \phi_{e}^{\prime}(y)|-| \phi_{e}^{\prime}(x)|| \leq L \inf _{u \in W_{t(e)}}\left|\phi_{e}^{\prime}(u)\right|\|y-x\|^{\alpha} .
$$

The associated geometric potential $\zeta_{\Phi}: \Sigma_{\Phi} \rightarrow \mathbb{R}^{-}$of a conformal GDMS $\Phi$ is defined by

$$
\zeta_{\Phi}(\omega):=\log \left|\phi_{\omega_{1}}^{\prime}\left(\pi_{\Phi}(\sigma(\omega))\right)\right|, \text { for all } \omega \in \Sigma_{\Phi}
$$

A Markov shift $\Sigma$ with a finite or countable alphabet $I$ is called finitely irreducible if there exists a finite set $\Lambda \subset \Sigma^{*}$ such that for all $i, j \in I$ there exists a word $\omega \in \Lambda \cup\{\varnothing\}$ such that $i \omega j \in \Sigma^{*}$ (see [MU03, Section 2]). Note that if $I$ is finite, then $\Sigma$ is finitely irreducible if and only if $\Sigma$ is irreducible. The following result from [RU08, Theorem 3.7] shows that in the sense of Hausdorff dimension, the limit set of a conformal GDMS with a finitely irreducible incidence matrix can be exhausted by its finitely generated subsystems. The last equality in Theorem 2.9 follows from [JKL14, Corollary 2.10] since the associated geometric potential of the conformal GDMS $\Phi$ is bounded away from zero by $-\log (s)$, where $s$ denotes the uniform bound of the Lipschitz constants of the contractions of $\Phi$ (see Definition 2.7). 
Theorem 2.9 (Generalized Bowen's formula). Let $\Phi$ be a conformal GDMS such that $\Sigma_{\Phi}$ is finitely irreducible. We then have that

$$
\operatorname{dim}_{H}(J(\Phi))=\operatorname{dim}_{H}\left(J^{*}(\Phi)\right)=\inf \left\{s \in \mathbb{R}: \mathcal{P}\left(s \zeta_{\Phi}\right) \leq 0\right\}=\mathcal{P}_{-\zeta_{\Phi}}\left(0, \Sigma_{\Phi}^{*}\right) .
$$

Let us now give the definition of a GDMS $\Phi$ associated to the free group $\mathbb{F}_{d}$ of rank $d \geq 2$ and introduce the radial limit set of a normal subgroup $N$ of $\mathbb{F}_{d}$ with respect to $\Phi$.

Definition 2.10. Let $\Phi=\left(V,\left(X_{v}\right)_{v \in V}, E, i, t,\left(\phi_{e}\right)_{e \in E}, A\right)$ be a GDMS and let $d \geq 2$. The GDMS $\Phi$ is said to be associated to $\mathbb{F}_{d}=\left\langle g_{1}, \ldots, g_{d}\right\rangle$ if $V=$ $\left\{g_{1}, g_{1}^{-1}, \ldots, g_{d}, g_{d}^{-1}\right\}, E=\left\{(v, w) \in V^{2}: v \neq w^{-1}\right\}$, the maps $i, t: E \rightarrow V$ are given by $i(v, w)=v$ and $t(v, w)=w$, for each $(v, w) \in E$, and the incidence matrix $A=(a(e, f)) \in\{0,1\}^{E \times E}$ satisfies $a(e, f)=1$ if and only if $t(e)=i(f)$, for all $e, f \in E$. If additionally $\Phi$ is a conformal GDMS such that, for each $(v, w) \in E$, the map $\phi_{(v, w)}$ is a similarity for which the contraction ratio is independent of $w$, then $\Phi$ is called a linear GDMS associated to $\mathbb{F}_{d}$.

For a subgroup $H$ of $\mathbb{F}_{d}$ and a GDMS $\Phi$ associated to $\mathbb{F}_{d}$, the radial and the uniformly radial limit set of $H$ with respect to $\Phi$ are respectively given by

$$
\Lambda_{\mathrm{r}}(H, \Phi):=\pi_{\Phi}\left\{\left(v_{i}, w_{i}\right) \in \Sigma_{\Phi}: \exists \gamma \in \mathbb{F}_{d} \text { such that for infinitely many } n \in \mathbb{N},\right.
$$

$$
\left.v_{1} \ldots v_{n} \in H \gamma\right\}
$$

and

$\Lambda_{\mathrm{ur}}(H, \Phi):=\pi_{\Phi}\left\{\left(v_{i}, w_{i}\right) \in \Sigma_{\Phi}: \exists \Gamma \subset \mathbb{F}_{d}\right.$ finite, such that for all $n \in \mathbb{N}$,

$$
\left.v_{1} \ldots v_{n} \in H \Gamma\right\} .
$$

Remark. It is clear that if $\Phi$ is a GDMS generated by a family of similarity maps, then $\Phi$ automatically satisfies (c) and (e) in Definition 2.8 of a conformal GDMS.

2.3. Random walks on graphs and amenability. In this section we collect some useful definitions and results concerning random walks on graphs. We will mainly follow Woe00.

Definition 2.11. A graph $X=(V, E)$ consists of a countable vertex set $V$ and an edge set $E \subset V \times V$ such that $(v, w) \in E$ if and only if $(w, v) \in E$. We write $v \sim w$ if $(v, w) \in E$, which defines an equivalence relation on $V$. For all $v, w \in V$ and $k \in \mathbb{N}_{0}$, a path of length $k$ from $v$ to $w$ is a sequence $\left(v_{0}, \ldots, v_{k}\right) \in V^{k+1}$ such that $v_{0}=v, v_{k}=w$ and $v_{i-1} \sim v_{i}$ for all $1 \leq i \leq k$. For all $v \in V$, let $\operatorname{deg}(v):=\operatorname{card}\{w \in V: w \sim v\}$ denote the degree of the vertex $v$. The graph $(V, E)$ is called connected if for all $v, w \in V$ with $v \neq w$, there exists $k \in \mathbb{N}$ and a path of length $k$ from $v$ to $w$. For a connected graph $X=(V, E)$ and $v, w \in V$ we let $d_{X}(v, w)$ denote the minimal length of all paths from $v$ to $w$, which defines the graph metric $d_{X}(\cdot, \cdot): V \times V \rightarrow \mathbb{N}_{0}$. The graph $(V, E)$ is said to have bounded geometry if it is connected and if $\sup _{v \in V}\{\operatorname{deg}(v)\}<\infty$. For each set of vertices $A \subset V$ we define $d A:=\{v \in A: \exists w \in V \backslash A$ such that $v \sim w\}$.

We now recall an important property of groups, which was introduced by von Neumann [Neu29] under the German name messbar. Later, groups with this property were renamed amenable groups by Day Day49] and also referred to as groups with full Banach mean value by Følner Føl55. 
Definition 2.12. A discrete group $G$ is said to be amenable if there exists a finitely additive probability measure $\nu$ on the set of all subsets of $G$ which is invariant under left multiplication by elements of $G$, that is, $\nu(A)=\nu(g(A))$ for all $g \in G$ and $A \subset G$.

We will also require the concept of an amenable graph, which extends the concept of amenability for groups (see Proposition 2.17 below).

Definition 2.13. A graph $X=(V, E)$ with bounded geometry is called amenable if and only if there exists $\kappa>0$ such that for all finite sets $A \subset V$ we have $\operatorname{card}(A) \leq \kappa \operatorname{card}(d A)$.

For the study of graphs in terms of amenability, the following definition is useful.

Definition 2.14. A rough isometry (or quasi-isometry) between two metric spaces $\left(Y, d_{Y}\right)$ and $\left(Y^{\prime}, d_{Y^{\prime}}\right)$ is a map $\varphi: Y \rightarrow Y^{\prime}$ which has the following properties. There exist constants $A, B>0$ such that for all $y_{1}, y_{2} \in Y$ we have

$$
A^{-1} d_{Y}\left(y_{1}, y_{2}\right)-A^{-1} B \leq d_{Y^{\prime}}\left(\varphi\left(y_{1}\right), \varphi\left(y_{2}\right)\right) \leq A d_{Y}\left(y_{1}, y_{2}\right)+B
$$

and for all $y^{\prime} \in Y^{\prime}$ we have

$$
d_{Y^{\prime}}\left(y^{\prime}, \varphi(Y)\right) \leq B .
$$

Two metric spaces $\left(Y, d_{Y}\right)$ and $\left(Y^{\prime}, d_{Y^{\prime}}\right)$ are said to be roughly isometric if there exists a rough isometry between $\left(Y, d_{Y}\right)$ and $\left(Y^{\prime}, d_{Y^{\prime}}\right)$. For connected graphs $X=$ $(V, E)$ and $X=\left(V^{\prime}, E^{\prime}\right)$ with graph metrics $d_{X}$ and $d_{X^{\prime}}$, we say that the graphs $X$ and $X^{\prime}$ are roughly isometric if the metric spaces $\left(V, d_{X}\right)$ and $\left(V^{\prime}, d_{X^{\prime}}\right)$ are roughly isometric.

The next theorem states that amenability of graphs is invariant under rough isometries ([Woe00, Theorem 4.7]).

Theorem 2.15. Let $X$ and $X^{\prime}$ be graphs with bounded geometry such that $X$ and $X^{\prime}$ are roughly isometric. We then have that $X$ is amenable if and only if $X^{\prime}$ is amenable.

The Cayley graph of a group provides the connection between groups and graphs.

Definition 2.16. We say that a set $S \subset G$ is a symmetric set of generators of the group $G$ if $\langle S\rangle=G$ and if $g^{-1} \in S$, for all $g \in S$. For a group $G$ and a symmetric set of generators $S$, the Cayley graph of $G$ with respect to $S$ is the graph with vertex set $G$ and edge set $E:=\left\{\left(g, g^{\prime}\right) \in G \times G: g^{-1} g^{\prime} \in S\right\}$. We denote this graph by $X(G, S)$.

The next proposition shows that amenability of groups and graphs is compatible ([Woe00, Proposition 12.4]).

Proposition 2.17. A finitely generated group $G$ is amenable if and only if one (and hence every) Cayley graph $X(G, S)$ of $G$ with respect to a finite symmetric set of generators $S \subset G$ is amenable.

Let us now relate amenability of graphs to spectral properties of transition operators.

Definition 2.18. For a finite or countably infinite discrete vertex set $V$, we say that the matrix $P=(p(v, w)) \in \mathbb{R}^{V \times V}$ is a transition matrix on $V$ if $p(v, w) \geq 0$ and $\sum_{u \in V} p(v, u)=1$, for all $v, w \in V$. A Borel measure $\nu$ supported on $V$ is $P$-invariant if $\sum_{u \in V} \nu(u) p(u, w)=\nu(w)$, for all $w \in V$. 
The following definitions introduce the concept of a transition matrix to be adapted to a graph (see [Woe00, (1.20, 1.21)]).

Definition 2.19. For a connected graph $X=(V, E)$ and a transition matrix $P=(p(v, w)) \in \mathbb{R}^{V \times V}$ on $V$, we say that $P$ is uniformly irreducible with respect to $X$ if there exist $K \in \mathbb{N}$ and $\epsilon>0$ such that for all $v, w \in V$ satisfying $v \sim w$, there exists $k \in \mathbb{N}$ with $k \leq K$ such that $p^{(k)}(v, w) \geq \epsilon$. We say that $P$ has bounded range with respect to $X$ if there exists $R>0$ such that $p(v, w)=0$ whenever $d_{X}(v, w)>R$.

Let $P=(p(v, w)) \in \mathbb{R}^{V \times V}$ be a transition matrix on $V$ with a $P$-invariant Borel measure $\nu$ on $V$. It is well known that $P$ defines a linear operator on $\ell^{2}(V, \nu)$ through the equations

$$
P f(v):=\sum_{w \in V} p(v, w) f(w), \quad \text { for all } v \in V \text { and } f \in \ell^{2}(V, \nu),
$$

and that the norm of this operator is less than or equal to one. For the spectral radius $\rho(P)$ of the operator $P$ on $\ell^{2}(V, \nu)$ we cite the following result from OW07. This result has a rather long history going back to Kes59b, Kes59a (see also Day64, Coh82,Dod84,DK86, Ger88, Moh88, Kai92,Woe00]).

Theorem 2.20 (Ortner, Woess). Let $X=(V, E)$ be a graph with bounded geometry and let $P$ denote a transition matrix on $V$ such that $P$ is uniformly irreducible with respect to $X$ and has bounded range with respect to $X$. If there exists a $P$-invariant Borel measure $\nu$ on $V$ and a constant $C \geq 1$ such that $C^{-1} \leq \nu(w) \leq C$, for all $w \in V$, then we have that $\rho(P)=1$ if and only if $X$ is amenable.

\section{Thermodynamic Formalism For Group-eXtended MARKov Systems}

Throughout this section our setting is as follows.

(1) $\Sigma$ is a Markov shift with finite alphabet $I$ and left-shift map $\sigma: \Sigma \rightarrow \Sigma$.

(2) $G$ is a countable discrete group $G$ with Haar measure (counting measure) $\lambda$.

(3) $\Psi: I^{*} \rightarrow G$ is a semigroup homomorphism such that the following property holds. For all $a, b \in I$ there exists $\gamma \in \Psi^{-1}\{\mathrm{id}\} \cap \Sigma^{*} \cup\{\varnothing\}$ such that $a \gamma b \in \Sigma^{*}$.

(4) $\varphi: \Sigma \rightarrow \mathbb{R}$ denotes a Hölder continuous potential with $\sigma$-invariant Gibbs measure $\mu_{\varphi}, \mathcal{L}_{\varphi}: C_{b}(\Sigma) \rightarrow C_{b}(\Sigma)$ denotes the Perron-Frobenius operator associated to $\varphi$, and $h: \Sigma \rightarrow \mathbb{R}$ denotes the unique Hölder continuous eigenfunction of $\mathcal{L}_{\varphi}$ with corresponding eigenvalue $\mathrm{e}^{\mathcal{P}(\varphi)}$ whose existence is guaranteed by Theorem 2.6 .

In this section we will address the following problem.

Problem 3.1. How is amenability of $G$ reflected in the relationship between the quantities $\mathcal{P}\left(\varphi, \Psi^{-1}\{\right.$ id $\left.\} \cap \Sigma^{*}\right)$ and $\mathcal{P}(\varphi)$ ?

It turns out that in order to investigate Problem 3.1 it is helpful to consider group-extended Markov systems (see Definition 3.2), which were studied in AD00, AD02 for certain abelian groups. 
Definition 3.2. The skew-product dynamics on $(\Sigma \times G, \sigma \rtimes \Psi)$, for which the transformation $\sigma \rtimes \Psi: \Sigma \times G \rightarrow \Sigma \times G$ is given by

$$
(\sigma \rtimes \Psi)(\omega, g):=\left(\sigma(\omega), g \Psi\left(\omega_{1}\right)\right), \text { for all }(\omega, g) \in \Sigma \times G,
$$

is called a group-extended Markov system. We let $\pi_{1}: \Sigma \times G \rightarrow \Sigma$ and $\pi_{2}: \Sigma \times G \rightarrow$ $G$ denote the projections to the first and second factors of $\Sigma \times G$.

Remark. Throughout, we assume that $\Sigma \times G$ is equipped with the product topology. Note that by item (3) of our standing assumptions we have that the group-extended Markov system $(\Sigma \times G, \sigma \rtimes \Psi)$ is topologically transitive if and only if $\Psi\left(\Sigma^{*}\right)=G$.

3.1. Perron-Frobenius theory. In this section, we investigate the relationship between the pressure $\mathcal{P}\left(\varphi, \Psi^{-1}\{\mathrm{id}\} \cap \Sigma^{*}\right)$ and the spectral radius of a PerronFrobenius operator associated to $(\Sigma \times G, \sigma \rtimes \Psi)$, which will be introduced in Definition 3.4 below. Combining this with results concerning transition operators of random walks on graphs, which will be given in Section 3.2, we are able to give a complete answer to Problem 3.1 for potentials $\varphi$ depending only on a finite number of coordinates (see Theorem 3.21).

Let us begin by stating the following lemma. The proof is straightforward and is thus left to the reader.

Lemma 3.3. The measure $\mu_{\varphi} \times \lambda$ is $(\sigma \rtimes \Psi)$-invariant.

Next, we define the Koopman operator ([Koo31,LM94] ) and the Perron-Frobenius operator associated to the group-extended Markov system $(\Sigma \times G, \sigma \rtimes \Psi)$. Note that the previous lemma ensures that these operators are well defined. We denote by $L^{2}\left(\Sigma \times G, \mu_{\varphi} \times \lambda\right)$ the Hilbert space of real-valued functions on $\Sigma \times G$ which are square-integrable with respect to $\mu_{\varphi} \times \lambda$.

Definition 3.4. The Koopman operator

$$
U: L^{2}\left(\Sigma \times G, \mu_{\varphi} \times \lambda\right) \rightarrow L^{2}\left(\Sigma \times G, \mu_{\varphi} \times \lambda\right)
$$

is given by

$$
U(f):=f \circ(\sigma \rtimes \Psi),
$$

and the Perron-Frobenius operator

$$
\mathcal{L}_{\varphi \circ \pi_{1}}: L^{2}\left(\Sigma \times G, \mu_{\varphi} \times \lambda\right) \rightarrow L^{2}\left(\Sigma \times G, \mu_{\varphi} \times \lambda\right)
$$

is given by

$$
\mathcal{L}_{\varphi \circ \pi_{1}}:=\mathrm{e}^{\mathcal{P}(\varphi)} M_{h \circ \pi_{1}} \circ U^{*} \circ\left(M_{h \circ \pi_{1}}\right)^{-1},
$$

where the multiplication operator

$$
M_{h \circ \pi_{1}}: L^{2}\left(\Sigma \times G, \mu_{\varphi} \times \lambda\right) \rightarrow L^{2}\left(\Sigma \times G, \mu_{\varphi} \times \lambda\right)
$$

is given by

$$
M_{h \circ \pi_{1}}(f):=f \cdot\left(h \circ \pi_{1}\right)
$$

and $U^{*}: L^{2}\left(\Sigma \times G, \mu_{\varphi} \times \lambda\right) \rightarrow L^{2}\left(\Sigma \times G, \mu_{\varphi} \times \lambda\right)$ denotes the adjoint of $U$.

The proof of the next lemma is straightforward and therefore omitted.

Lemma 3.5. The following properties hold for the bounded linear operators $U, \mathcal{L}_{\varphi \circ \pi_{1}}: L^{2}\left(\Sigma \times G, \mu_{\varphi} \times \lambda\right) \rightarrow L^{2}\left(\Sigma \times G, \mu_{\varphi} \times \lambda\right):$

(1) $U$ is an isometry, so we have that $\|U\|=\rho(U)=1$, where $\rho$ denotes the spectral radius of $U$. 
(2) For $f \in L^{2}\left(\Sigma \times G, \mu_{\varphi} \times \lambda\right)$ and $\left(\mu_{\varphi} \times \lambda\right)$-almost every $(\omega, g) \in \Sigma \times G$ we have that

$$
\mathcal{L}_{\varphi \circ \pi_{1}}(f)(\omega, g)=\sum_{i \in I: i \omega_{1} \in \Sigma^{2}} \mathrm{e}^{\varphi(i \omega)} f\left(i \omega, g \Psi(i)^{-1}\right) .
$$

(3) For the spectral radius of $\mathcal{L}_{\varphi \circ \pi_{1}}$ we obtain that $\rho\left(\mathcal{L}_{\varphi \circ \pi_{1}}\right)=\mathrm{e}^{\mathcal{P}(\varphi)}$.

Remark. The representation of $\mathcal{L}_{\varphi \circ \pi_{1}}$ in Lemma 3.5 (2) extends Definition 2.5 of the Perron-Frobenius operator for Markov shifts with a finite alphabet.

The next lemma gives relationships between $\mathcal{P}\left(\varphi, \Psi^{-1}\{\right.$ id $\left.\} \cap \Sigma^{*}\right)$ and $\mathcal{L}_{\varphi \circ \pi_{1}}$. Before stating the lemma, let us fix some notation. We write $\mathbb{1}_{A}$ for the characteristic function of a set $A$ and we use $\left\{\pi_{2}=g\right\}$ to denote the set $\pi_{2}^{-1}\{g\}$, for each $g \in G$. Further, let $\mathcal{B}(\Sigma \times G)$ denote the Borel $\sigma$-algebra on $\Sigma \times G$.

Lemma 3.6. For all sets $A, B \in \mathcal{B}(\Sigma \times G)$ and for each $n \in \mathbb{N}$ we have that

$$
\begin{aligned}
\frac{\min h}{\max h} \mu_{\varphi}\left(A \cap(\sigma \rtimes \Psi)^{-n}(B)\right) & \leq \mathrm{e}^{-n \mathcal{P}(\varphi)}\left(\mathcal{L}_{\varphi \circ \pi_{1}}^{n}\left(\mathbb{1}_{A}\right), \mathbb{1}_{B}\right) \\
& \leq \frac{\max h}{\min h} \mu_{\varphi}\left(A \cap(\sigma \rtimes \Psi)^{-n}(B)\right) .
\end{aligned}
$$

Moreover, for all $g, g^{\prime} \in G$ we have that

$$
\limsup _{n \rightarrow \infty} \frac{1}{n} \log \left(\mathcal{L}_{\varphi \circ \pi_{1}}^{n}\left(\mathbb{1}_{\left\{\pi_{2}=g\right\}}\right), \mathbb{1}_{\left\{\pi_{2}=g^{\prime}\right\}}\right)=\mathcal{P}\left(\varphi, \Psi^{-1}\left\{g^{-1} g^{\prime}\right\} \cap \Sigma^{*}\right) .
$$

Proof. For the first assertion, observe that by the definition of $\mathcal{L}_{\varphi \circ \pi_{1}}$ we have that

$$
\begin{aligned}
\left(\mathcal{L}_{\varphi \circ \pi_{1}}^{n}\left(\mathbb{1}_{A}\right), \mathbb{1}_{B}\right) & =\mathrm{e}^{n \mathcal{P}(\varphi)}\left(M_{h \circ \pi_{1}} \circ\left(U^{*}\right)^{n} \circ\left(M_{h \circ \pi_{1}}\right)^{-1}\left(\mathbb{1}_{A}\right), \mathbb{1}_{B}\right) \\
& =\mathrm{e}^{n \mathcal{P}(\varphi)}\left(\left(M_{h \circ \pi_{1}}\right)^{-1}\left(\mathbb{1}_{A}\right),\left(M_{h \circ \pi_{1}}\left(\mathbb{1}_{B}\right)\right) \circ(\sigma \rtimes \Psi)^{n}\right) .
\end{aligned}
$$

Since the continuous function $h: \Sigma \rightarrow \mathbb{R}^{+}$is bounded away from zero and infinity on the compact set $\Sigma$, the first assertion follows.

The second assertion follows from the first if we set $A:=\left\{\pi_{2}=g\right\}$ and $B:=$ $\left\{\pi_{2}=g^{\prime}\right\}$ and use the Gibbs property (2.1) of $\mu_{\varphi}$.

As an immediate consequence of the previous lemma, we obtain the following upper bound for $\mathcal{P}\left(\varphi, \Psi^{-1}\left\{g^{-1} g^{\prime}\right\} \cap \Sigma^{*}\right)$ in terms of the spectral radius of $\mathcal{L}_{\varphi \circ \pi_{1}}$.

Corollary 3.7. Let $V \subset L^{2}\left(\Sigma \times G, \mu_{\varphi} \times \lambda\right)$ be a closed $\mathcal{L}_{\varphi \circ \pi_{1}}$-invariant linear subspace such that $\mathbb{1}_{\left\{\pi_{2}=g\right\}}, \mathbb{1}_{\left\{\pi_{2}=g^{\prime}\right\}} \in V$, for some $g, g^{\prime} \in G$. We then have that

$$
\mathcal{P}\left(\varphi, \Psi^{-1}\left\{g^{-1} g^{\prime}\right\} \cap \Sigma^{*}\right) \leq \log \rho\left(\left.\mathcal{L}_{\varphi \circ \pi_{1}}\right|_{V}\right) .
$$

Proof. By the Cauchy-Schwarz inequality and Gelfand's formula for the spectral radius ([Rud73, Theorem 10.13]), we have that

$$
\begin{aligned}
\limsup _{n \rightarrow \infty} \frac{1}{n} \log \left(\left.\mathcal{L}_{\varphi \circ \pi_{1}}^{n}\right|_{V}\left(\mathbb{1}_{\left\{\pi_{2}=g\right\}}\right), \mathbb{1}_{\left\{\pi_{2}=g^{\prime}\right\}}\right) & \leq \limsup _{n \rightarrow \infty} \frac{1}{n} \log \left\|\left.\mathcal{L}_{\varphi \circ \pi_{1}}^{n}\right|_{V}\right\| \\
& =\log \rho\left(\left.\mathcal{L}_{\varphi \circ \pi_{1}}\right|_{V}\right) .
\end{aligned}
$$

Combining the above inequality with the second assertion of Lemma 3.6 completes the proof. 
Recall that for a closed linear subspace $V \subset L^{2}\left(\Sigma \times G, \mu_{\varphi} \times \lambda\right)$, a bounded linear operator $T: V \rightarrow V$ is called positive if $T\left(V^{+}\right) \subset V^{+}$, where the positive cone $V^{+}$is defined by $V^{+}:=\{f \in V: f \geq 0\}$.

The following lemma will be crucial in order to obtain equality in the inequality stated in Corollary 3.7. The lemma extends a result of Gerl (see Ger88, and also Woe00, Lemma 10.1]).

Lemma 3.8. Let $V$ be a closed linear subspace of $L^{2}\left(\Sigma \times G, \mu_{\varphi} \times \lambda\right)$ and suppose that $\left\{\mathbb{1}_{\left\{\pi_{2}=g\right\}}: g \in G\right\} \subset V$. Let $T: V \rightarrow V$ be a self-adjoint bounded linear operator on $V$, which is positive and which satisfies $\operatorname{ker}(T) \cap V^{+}=\{0\}$. We then have that

$$
\sup _{g, g^{\prime} \in G}\left\{\limsup _{n \rightarrow \infty}\left|\left(T^{n}\left(\mathbb{1}_{\left\{\pi_{2}=g\right\}}\right), \mathbb{1}_{\left\{\pi_{2}=g^{\prime}\right\}}\right)\right|^{1 / n}\right\}=\|T\|=\rho(T) .
$$

Proof. Since $T$ is self-adjoint, it follows that $\|T\|=\rho(T)$. As in the proof of Corollary 3.7. one immediately verifies that

$$
\sup _{g, g^{\prime} \in G}\left\{\limsup _{n \rightarrow \infty}\left|\left(T^{n}\left(\mathbb{1}_{\left\{\pi_{2}=g\right\}}\right), \mathbb{1}_{\left\{\pi_{2}=g^{\prime}\right\}}\right)\right|^{1 / n}\right\} \leq \rho(T) .
$$

Let us first give an outline for the proof of the opposite inequality. We will first prove that for all $f \in V^{+}$with $f \neq 0$, the sequence $\left(\left(T^{n+1} f, T^{n+1} f\right) /\left(T^{n} f, T^{n} f\right)\right)_{n \in \mathbb{N}_{0}}$ is non-decreasing. This will then imply that the following limits exist and are equal:

$$
\lim _{n \rightarrow \infty} \frac{\left(T^{n+1} f, T^{n+1} f\right)}{\left(T^{n} f, T^{n} f\right)}=\lim _{n \rightarrow \infty}\left(T^{n} f, T^{n} f\right)^{1 / n} .
$$

From this we obtain for every $f \in V^{+}$with $f \neq 0$ that

$$
\frac{(T f, T f)}{(f, f)} \leq \lim _{n \rightarrow \infty}\left(T^{n} f, T^{n} f\right)^{1 / n} .
$$

Subsequently, we make use of the fact that

$$
\begin{aligned}
D^{\prime}:=\left\{f \in L^{2}\left(\Sigma \times G, \mu_{\varphi} \times \lambda\right) \cap L^{\infty}\left(\Sigma \times G, \mu_{\varphi} \times \lambda\right)\right. & :\left.f\right|_{\left\{\pi_{2}=g\right\}}=0 \\
& \text { for almost every } g \in G\}
\end{aligned}
$$

is dense in $L^{2}\left(\Sigma \times G, \mu_{\varphi} \times \lambda\right)$ and hence $D:=D^{\prime} \cap V$ is dense in $V$. For $f \in D$ we show that

$$
\lim _{n \rightarrow \infty}\left(T^{n} f, T^{n} f\right)^{1 / n} \leq \sup _{g, g^{\prime} \in G}\left\{\limsup _{n \rightarrow \infty}\left|\left(T^{2 n}\left(\mathbb{1}_{\left\{\pi_{2}=g\right\}}\right), \mathbb{1}_{\left\{\pi_{2}=g^{\prime}\right\}}\right)\right|^{1 / n}\right\} .
$$

Combining this with (3.2) applied to $|f|$, we conclude for $f \in D$ with $f \neq 0$ that (3.3)

$$
\frac{(T f, T f)}{(f, f)} \leq \frac{(T|f|, T|f|)}{(|f|,|f|)} \leq \sup _{g, g^{\prime} \in G}\left\{\limsup _{n \rightarrow \infty}\left|\left(T^{2 n}\left(\mathbb{1}_{\left\{\pi_{2}=g\right\}}\right), \mathbb{1}_{\left\{\pi_{2}=g^{\prime}\right\}}\right)\right|^{1 / n}\right\} .
$$

Since $D$ is dense in $V$, there exists a sequence $\left(f_{n}\right)_{n \in \mathbb{N}} \in D^{\mathbb{N}}$ such that $\lim _{n}\left(T f_{n}, T f_{n}\right)$ $=\|T\|$ and $\left(f_{n}, f_{n}\right)=1$, for each $n \in \mathbb{N}$. Combining this observation with the estimate in (3.3), we conclude that

$$
\|T\| \leq \sup _{g, g^{\prime} \in G}\left\{\limsup _{n}\left|\left(T^{2 n}\left(\mathbb{1}_{\left\{\pi_{2}=g\right\}}\right), \mathbb{1}_{\left\{\pi_{2}=g^{\prime}\right\}}\right)\right|^{1 / 2 n}\right\} .
$$


Let us now turn to the details. We first verify that for every $f \in V^{+}$with $f \neq 0$, the sequence $\left(a_{n}\right)_{n \in \mathbb{N}_{0}}$ of positive real numbers, given for $n \in \mathbb{N}_{0}$ by $a_{n}:=$ $\left(T^{n+1} f, T^{n+1} f\right) /\left(T^{n} f, T^{n} f\right)$, is non-decreasing. Using that $T$ is self-adjoint and applying the Cauchy-Schwarz inequality, we have for $n \in \mathbb{N}_{0}$ that

$$
\left(T^{n+1} f, T^{n+1} f\right)^{2}=\left(T^{n} f, T^{n+2} f\right)^{2} \leq\left(T^{n} f, T^{n} f\right)\left(T^{n+2} f, T^{n+2} f\right) .
$$

Since $\left(T^{n} f, T^{n} f\right) \neq 0$ for all $n \in \mathbb{N}_{0}$ by our hypothesis, we can multiply both sides of (3.4) by $\left(T^{n+1} f, T^{n+1} f\right)^{-1}\left(T^{n+2} f, T^{n+2} f\right)^{-1}$, which proves that $\left(a_{n}\right)_{n \in \mathbb{N}_{0}}$ is non-decreasing. Hence, we have that $\lim _{n \rightarrow \infty} a_{n} \in \mathbb{R}^{+} \cup\{\infty\}$ exists. Observing that $\log \left(T^{n} f, T^{n} f\right)$ is equal to the telescoping sum $\log (f, f)+\sum_{j=0}^{n-1} \log a_{j}$ and using that $\lim _{n \rightarrow \infty} \log \left(a_{n}\right)$ is equal to its Cesàro mean, we deduce that

$$
\lim _{n \rightarrow \infty} \frac{1}{n} \log \left(T^{n} f, T^{n} f\right)=\lim _{n \rightarrow \infty} \frac{1}{n} \log (f, f)+\lim _{n \rightarrow \infty} \frac{1}{n} \sum_{j=0}^{n-1} \log a_{j}=\lim _{n \rightarrow \infty} \log a_{n},
$$

which proves (3.1). Since $\left(T^{n} f, T^{n} f\right)^{1 / n} \leq\|T\|^{2} \max \left\{\|f\|_{2}^{2}, 1\right\}$, for all $n \in \mathbb{N}$, we have that the limits in (3.1) are both finite.

It remains to prove that (3.3) holds for every $f \in D$ with $f \neq 0$. By definition of $D$, there exists a finite set $G_{0} \subset G$ such that $f=\sum_{g \in G_{0}} f \mathbb{1}_{\left\{\pi_{2}=g\right\}}$. Since $T$ is positive and self-adjoint, we conclude that

$$
\begin{aligned}
\left(T^{n} f, T^{n} f\right) \leq & \left(T^{n}|f|, T^{n}|f|\right)=\left(T^{2 n}|f|,|f|\right) \\
& =\sum_{g, g^{\prime} \in G_{0}}\left(T^{2 n}\left|f \mathbb{1}_{\left\{\pi_{2}=g\right\}}\right|,\left|f \mathbb{1}_{\left\{\pi_{2}=g^{\prime}\right\}}\right|\right) \\
\leq & \sum_{g, g^{\prime} \in G_{0}}\|f\|_{L^{\infty}\left(\Sigma \times G, \mu_{\varphi} \times \lambda\right)}\left(T^{2 n} \mathbb{1}_{\left\{\pi_{2}=g\right\}}, \mathbb{1}_{\left\{\pi_{2}=g^{\prime}\right\}}\right) .
\end{aligned}
$$

Finally, raising both sides of the previous inequality to the power $1 / n$ and letting $n$ tend to infinity gives

$$
\lim _{n \rightarrow \infty}\left(T^{n} f, T^{n} f\right)^{1 / n} \leq \max _{g, g^{\prime} \in G_{0}} \limsup _{n \rightarrow \infty}\left|\left(T^{2 n} \mathbb{1}_{\left\{\pi_{2}=g\right\}}, \mathbb{1}_{\left\{\pi_{2}=g^{\prime}\right\}}\right)\right|^{1 / n},
$$

and the estimate in (3.3) follows. The proof is complete.

Regarding the requirements of the previous proposition, we prove the following for $\mathcal{L}_{\varphi \circ \pi_{1}}$.

Lemma 3.9. Let $V$ be a closed $\mathcal{L}_{\varphi \circ \pi_{1}}$-invariant linear subspace of $L^{2}\left(\Sigma \times G, \mu_{\varphi} \times \lambda\right)$ and suppose that $\mathcal{L}_{\varphi} \mathbb{1}=\mathbb{1}$. Then, $\left.\mathcal{L}_{\varphi \circ \pi_{1}}\right|_{V}$ is a positive operator for which $\operatorname{ker}\left(\left.\mathcal{L}_{\varphi \circ \pi_{1}}\right|_{V}\right) \cap V^{+}=\{0\}$. Further, if $\left\{f^{-}: f \in V\right\} \subset V$, then $\left(\left.\mathcal{L}_{\varphi \circ \pi_{1}}\right|_{V}\right)^{*}$ is a positive operator, and if there exists $g \in V$ with $g>0$, then we have that $\operatorname{ker}\left(\left(\left.\mathcal{L}_{\varphi \circ \pi_{1}}\right|_{V}\right)^{*}\right) \cap V^{+}=\{0\}$.

Proof. Clearly, by definition of $\mathcal{L}_{\varphi \circ \pi_{1}}$, we have that $\left.\mathcal{L}_{\varphi \circ \pi_{1}}\right|_{V}$ is positive. Now let $f \in \operatorname{ker}\left(\left.\mathcal{L}_{\varphi \circ \pi_{1}}\right|_{V}\right) \cap V^{+}$. Since $\mu_{\varphi}$ is a fixed point of $\mathcal{L}_{\varphi}^{*}$, one deduces by the monotone convergence theorem and by the definition of $\mathcal{L}_{\varphi \circ \pi_{1}}$ that $\int f d\left(\mu_{\varphi} \times \lambda\right)=$ $\int \mathcal{L}_{\varphi \circ \pi_{1}}(f) d\left(\mu_{\varphi} \times \lambda\right)$. Hence, $f \in \operatorname{ker}\left(\left.\mathcal{L}_{\varphi \circ \pi_{1}}\right|_{V}\right) \cap V^{+}$implies $\int f d\left(\mu_{\varphi} \times \lambda\right)=0$, and so $f=0$. 
We now consider the adjoint operator $\left(\left.\mathcal{L}_{\varphi \circ \pi_{1}}\right|_{V}\right)^{*}$. Let $f \in V^{+}$. Since $\left\{f^{-}: f \in V\right\}$ $\subset V$ and using that $\mathcal{L}_{\varphi \circ \pi_{1}}$ is positive, we obtain that

$0 \geq\left(\left(\left.\mathcal{L}_{\varphi \circ \pi_{1}}\right|_{V}\right)^{*}(f),\left(\left(\left.\mathcal{L}_{\varphi \circ \pi_{1}}\right|_{V}\right)^{*}(f)\right)^{-}\right)=\left(f, \mathcal{L}_{\varphi \circ \pi_{1}}\left(\left(\left(\left.\mathcal{L}_{\varphi \circ \pi_{1}}\right|_{V}\right)^{*}(f)\right)^{-}\right)\right) \geq 0$.

Hence, we have

$$
\begin{aligned}
0 & =\left(\left(\left.\mathcal{L}_{\varphi \circ \pi_{1}}\right|_{V}\right)^{*}(f),\left(\left(\left.\mathcal{L}_{\varphi \circ \pi_{1}}\right|_{V}\right)^{*}(f)\right)^{-}\right) \\
& =-\left\|\left(\left(\left.\mathcal{L}_{\varphi \circ \pi_{1}}\right|_{V}\right)^{*}(f)\right)^{-}\right\|_{2}^{2},
\end{aligned}
$$

giving that $\left(\left.\mathcal{L}_{\varphi \circ \pi_{1}}\right|_{V}\right)^{*}$ is positive. Now let $f \in \operatorname{ker}\left(\left(\left.\mathcal{L}_{\varphi \circ \pi_{1}}\right|_{V}\right)^{*}\right) \cap V^{+}$be given and assume that there exists $g \in V$ with $g>0$. We then have that

$$
0=\left(\left(\left.\mathcal{L}_{\varphi \circ \pi_{1}}\right|_{V}\right)^{*}(f), g\right)=\left(f, \mathcal{L}_{\varphi \circ \pi_{1}}(g)\right) .
$$

Since $g>0$, we have $\mathcal{L}_{\varphi \circ \pi_{1}}(g)>0$, which implies that $f=0$. The proof is complete.

It turns out that the Perron-Frobenius operator is not self-adjoint in general. In fact, as we will see in the following remark, this operator is self-adjoint only in very special cases. Therefore, we will introduce the notion of an asymptotically self-adjoint operator in Definition 3.10 below.

Remark. We observe that the requirement that $\left.\mathcal{L}_{\varphi \circ \pi_{1}}\right|_{V}$ is self-adjoint, for some closed linear subspace $V$ of $L^{2}\left(\Sigma \times G, \mu_{\varphi} \times \lambda\right)$, is rather restrictive. Indeed, let $V$ be a closed linear subspace of $L^{2}\left(\Sigma \times G, \mu_{\varphi} \times \lambda\right)$ satisfying $\left\{\mathbb{1}_{[i] \times\{g\}}: i \in I, g \in G\right\}$ $\subset V$ and suppose that $\left.\mathcal{L}_{\varphi \circ \pi_{1}}\right|_{V}$ is self-adjoint. It follows that $j i \in \Sigma^{2}$ and $\Psi(i)$ $=\Psi(j)^{-1}$, for all $i, j \in I$ such that $i j \in \Sigma^{2}$. In particular, we have that $\Psi\left(\Sigma^{*}\right)$ has at most two elements. To prove this, let $i j \in \Sigma^{2}$ be given. By the Gibbs property (2.1) of $\mu_{\varphi}$ we have that $\mu_{\varphi}[i j]>0$. Setting $C:=\frac{\max h}{\min h} \mathrm{e}^{-\mathcal{P}(\varphi)}$ we deduce from Lemma 3.6 that

$$
\begin{aligned}
0 & <\left(\mu_{\varphi} \times \lambda\right)\left(([i] \times\{\mathrm{id}\}) \cap(\sigma \rtimes \Psi)^{-1}([j] \times\{\Psi(i)\})\right) \\
& \leq C\left(\left.\mathcal{L}_{\varphi \circ \pi_{1}}\right|_{V}\left(\mathbb{1}_{[i] \times\{\mathrm{id}\}}\right), \mathbb{1}_{[j] \times\{\Psi(i)\}}\right) .
\end{aligned}
$$

Using that $\left.\mathcal{L}_{\varphi \circ \pi_{1}}\right|_{V}$ is self-adjoint and again by Lemma 3.6. we conclude that

$$
\begin{aligned}
& \left(\left.\mathcal{L}_{\varphi \circ \pi_{1}}\right|_{V}\left(\mathbb{1}_{[i] \times\{\mathrm{id}\}}\right), \mathbb{1}_{[j] \times\{\Psi(i)\}}\right) \\
& \quad \leq C\left(\mu_{\varphi} \times \lambda\right)\left(([j] \times\{\Psi(i)\}) \cap(\sigma \rtimes \Psi)^{-1}([i] \times\{\mathrm{id}\})\right) .
\end{aligned}
$$

The previous two estimates imply that the set $([j] \times\{\Psi(i)\}) \cap(\sigma \rtimes \Psi)^{-1}([i] \times\{\mathrm{id}\})$ is non-empty. Hence, we have $j i \in \Sigma^{2}$ and $\Psi(i) \Psi(j)=\mathrm{id}$.

The next definition introduces a concept which is slightly weaker than selfadjointness.

Definition 3.10. Let $V$ be a closed linear subspace of $L^{2}\left(\Sigma \times G, \mu_{\varphi} \times \lambda\right)$ and let $T: V \rightarrow V$ be a positive bounded linear operator. We say that $T$ is asymptotically 
self-adjoint if there exist sequences $\left(c_{m}\right)_{m \in \mathbb{N}} \in\left(\mathbb{R}^{+}\right)^{\mathbb{N}}$ and $\left(N_{m}\right)_{m \in \mathbb{N}} \in \mathbb{N}_{0}^{\mathbb{N}}$, satisfying $\lim _{m \rightarrow \infty}\left(c_{m}\right)^{1 / m}=1$ and $\lim _{m \rightarrow \infty} m^{-1} N_{m}=0$, such that for all non-negative functions $f, g \in V$ and for all $n \in \mathbb{N}$ we have

$$
\left(T^{n} f, g\right) \leq c_{n} \sum_{i=0}^{N_{n}}\left(f, T^{n+i} g\right) .
$$

Remark. Note that $T$ is asymptotically self-adjoint if and only if $T^{*}$ is asymptotically self-adjoint. We also remark that it clearly suffices to verify (3.5) on a norm-dense subset of non-negative functions in $V$.

The next proposition shows that if $\left.\mathcal{L}_{\varphi \circ \pi_{1}}\right|_{V}$ is asymptotically self-adjoint, for some closed linear subspace $V$ of $L^{2}\left(\Sigma \times G, \mu_{\varphi} \times \lambda\right)$, then we can relate $\sup _{g \in G} \mathcal{P}\left(\varphi, \Psi^{-1}\{g\} \cap \Sigma^{*}\right)$ to the spectral radius of $\left.\mathcal{L}_{\varphi \circ \pi_{1}}\right|_{V}$. The proof, which makes use of Lemma 3.8 and Lemma 3.9, is inspired by OW07, Proposition 1.6].

Proposition 3.11. Suppose that $\mathcal{L}_{\varphi} \mathbb{1}=\mathbb{1}$ and let $V \subset L^{2}\left(\Sigma \times G, \mu_{\varphi} \times \lambda\right)$ be a closed linear $\mathcal{L}_{\varphi \circ \pi_{1}}$-invariant subspace such that $\left\{f^{-}: f \in V\right\} \subset V$ and $\left\{\mathbb{1}_{\left\{\pi_{2}=g\right\}}\right.$ : $g \in G\} \subset V$. If $\left.\mathcal{L}_{\varphi \circ \pi_{1}}\right|_{V}$ is asymptotically self-adjoint, then we have that

$$
\sup _{g \in G}\left\{\mathcal{P}\left(\varphi, \Psi^{-1}\{g\} \cap \Sigma^{*}\right)\right\}=\log \rho\left(\left.\mathcal{L}_{\varphi \circ \pi_{1}}\right|_{V}\right) .
$$

Proof. By Corollary 3.7, we have $\sup _{g \in G}\left\{\mathcal{P}\left(\varphi, \Psi^{-1}\{g\} \cap \Sigma^{*}\right)\right\} \leq \log \rho\left(\left.\mathcal{L}_{\varphi \circ \pi_{1}}\right|_{V}\right)$. Let us turn to the proof of the converse inequality. Using that $\left\|\left.\left(\left.\mathcal{L}_{\varphi \circ \pi_{1}}^{m}\right|_{V}\right)^{*} \mathcal{L}_{\varphi \circ \pi_{1}}^{m}\right|_{V}\right\|$ $=\left\|\left.\mathcal{L}_{\varphi \circ \pi_{1}}^{m}\right|_{V}\right\|^{2}$ for each $m \in \mathbb{N}$, it follows from Gelfand's formula ([Rud73, Theorem 10.13]) that

$$
\rho\left(\left.\mathcal{L}_{\varphi \circ \pi_{1}}\right|_{V}\right)=\lim _{n \rightarrow \infty}\left\|\left.\left(\left.\mathcal{L}_{\varphi \circ \pi_{1}}^{n}\right|_{V}\right)^{*} \mathcal{L}_{\varphi \circ \pi_{1}}^{n}\right|_{V}\right\|^{1 /(2 n)}
$$

Our next aim is to apply Lemma 3.8 to the self-adjoint operator

$$
T_{n}:=\left.\left(\left.\mathcal{L}_{\varphi \circ \pi_{1}}^{n}\right|_{V}\right)^{*} \mathcal{L}_{\varphi \circ \pi_{1}}^{n}\right|_{V},
$$

for each $n \in \mathbb{N}$. We have to verify that $T_{n}$ is positive and that $\operatorname{ker}\left(T_{n}\right) \cap V^{+}=\{0\}$, for each $n \in \mathbb{N}$. By Lemma 3.9. we have that $\left.\mathcal{L}_{\varphi \circ \pi_{1}}\right|_{V}$ is positive and $\operatorname{ker}\left(\left.\mathcal{L}_{\varphi \circ \pi_{1}}\right|_{V}\right) \cap$ $V^{+}=\{0\}$. Fix some arbitrary order for the elements in $G$, say $G=\left\{g_{i}: i \in \mathbb{N}\right\}$. Using that $V$ is a closed linear subspace containing $\left\{\mathbb{1}_{\left\{\pi_{2}=g_{i}\right\}}: i \in \mathbb{N}\right\}$, we obtain that $g:=\sum_{j \in \mathbb{N}} 2^{-j} \mathbb{1}_{\left\{\pi_{2}=g_{j}\right\}}>0$ is an element of $V$. Since $\left\{f^{-}: f \in V\right\} \subset V$ by our assumptions, Lemma 3.9 implies that $\left(\left.\mathcal{L}_{\varphi \circ \pi_{1}}\right|_{V}\right)^{*}$ is positive with $\operatorname{ker}\left(\left.\mathcal{L}_{\varphi \circ \pi_{1}}\right|_{V}\right)^{*} \cap$ $V^{+}=\{0\}$. Hence, for each $n \in \mathbb{N}$ we have that $T_{n}$ is positive and $\operatorname{ker}\left(T_{n}\right) \cap V^{+}=$ $\{0\}$. Consequently, it follows from Lemma 3.8 that for each $n \in \mathbb{N}$ we have

$$
\begin{aligned}
& \left\|\left.\left(\left.\mathcal{L}_{\varphi \circ \pi_{1}}^{n}\right|_{V}\right)^{*} \mathcal{L}_{\varphi \circ \pi_{1}}^{n}\right|_{V}\right\| \\
& \quad=\sup _{g, g^{\prime} \in G}\left\{\limsup _{k \rightarrow \infty}\left(\left(\left.\left(\left.\mathcal{L}_{\varphi \circ \pi_{1}}^{n}\right|_{V}\right)^{*} \mathcal{L}_{\varphi \circ \pi_{1}}^{n}\right|_{V}\right)^{k}\left(\mathbb{1}_{\left\{\pi_{2}=g\right\}}\right), \mathbb{1}_{\left\{\pi_{2}=g^{\prime}\right\}}\right)^{1 / k}\right\} .
\end{aligned}
$$

Let $g, g^{\prime} \in G$ be given. Using that $\left.\mathcal{L}_{\varphi \circ \pi_{1}}\right|_{V}$ is asymptotically self-adjoint, with sequences $\left(c_{m}\right)_{m \in \mathbb{N}} \in \mathbb{R}^{\mathbb{N}}$ and $\left(N_{m}\right)_{m \in \mathbb{N}} \in \mathbb{N}_{0}^{\mathbb{N}}$ as in Definition 3.10. we estimate for 
all $n \in \mathbb{N}$ that

$$
\begin{aligned}
& \limsup _{k \rightarrow \infty}\left(\left(\left.\left(\left.\mathcal{L}_{\varphi \circ \pi_{1}}^{n}\right|_{V}\right)^{*} \mathcal{L}_{\varphi \circ \pi_{1}}^{n}\right|_{V}\right)^{k}\left(\mathbb{1}_{\left\{\pi_{2}=g\right\}}\right), \mathbb{1}_{\left\{\pi_{2}=g^{\prime}\right\}}\right)^{1 / k} \\
\leq & \limsup _{k \rightarrow \infty}\left(c_{n}^{k} \sum_{i_{1}=0}^{N_{n}} \sum_{i_{2}=0}^{N_{n}} \cdots \sum_{i_{k}=0}^{N_{n}}\left(\left.\mathcal{L}_{\varphi \circ \pi_{1}}^{2 n k+\sum_{j=1}^{k} i_{j}}\right|_{V}\left(\mathbb{1}_{\left\{\pi_{2}=g\right\}}\right), \mathbb{1}_{\left\{\pi_{2}=g^{\prime}\right\}}\right)\right)^{1 / k} \\
\leq & c_{n} \limsup _{k \rightarrow \infty}\left(\left(N_{n}+1\right)^{k}\right. \\
\cdot & \left.\max _{\left(i_{1}, \ldots, i_{k}\right) \in\left\{0, \ldots, N_{n}\right\}^{k}}\left\{\left(\left.\mathcal{L}_{\varphi \circ \pi_{1}}^{2 n k+\sum_{j=1}^{k} i_{j}}\right|_{V}\left(\mathbb{1}_{\left\{\pi_{2}=g\right\}}\right), \mathbb{1}_{\left\{\pi_{2}=g^{\prime}\right\}}\right)\right\}\right)^{1 / k} .
\end{aligned}
$$

Let $\epsilon>0$. Since we have

$$
\limsup _{m \rightarrow \infty}\left(\left.\mathcal{L}_{\varphi \circ \pi_{1}}^{m}\right|_{V}\left(\mathbb{1}_{\left\{\pi_{2}=g\right\}}\right), \mathbb{1}_{\left\{\pi_{2}=g^{\prime}\right\}}\right)^{1 / m}=\mathrm{e}^{\mathcal{P}\left(\varphi, \Psi^{-1}\left\{g^{-1} g^{\prime}\right\} \cap \Sigma^{*}\right)}
$$

by Lemma 3.6, we obtain that

$$
\begin{array}{r}
\limsup _{k \rightarrow \infty}\left(\max _{\left(i_{1}, \ldots, i_{k}\right) \in\left\{0, \ldots, N_{n}\right\}^{k}}\left\{\left(\left.\mathcal{L}_{\varphi \circ \pi_{1}}^{2 n k+\sum_{j=1}^{k} i_{j}}\right|_{V}\left(\mathbb{1}_{\left\{\pi_{2}=g\right\}}\right), \mathbb{1}_{\left\{\pi_{2}=g^{\prime}\right\}}\right)\right\}\right)^{1 / k} \\
\leq \limsup _{k \rightarrow \infty}\left(\operatorname { m a x } _ { ( i _ { 1 } , \ldots , i _ { k } ) \in \{ 0 , \ldots , N _ { n } \} ^ { k } } \operatorname { m a x } \left\{\mathrm{e}^{\left(2 n k+k N_{n}\right)\left(\mathcal{P}\left(\varphi, \Psi^{-1}\left\{g^{-1} g^{\prime}\right\} \cap \Sigma^{*}\right)+\epsilon\right)}\right.\right. \\
\left.\left.\mathrm{e}^{2 n k\left(\mathcal{P}\left(\varphi, \Psi^{-1}\left\{g^{-1} g^{\prime}\right\} \cap \Sigma^{*}\right)+\epsilon\right)}\right\}\right)^{1 / k} .
\end{array}
$$

Since $\epsilon>0$ was chosen to be arbitrary, our previous estimates imply that for each $n \in \mathbb{N}$ and for all $g, g^{\prime} \in G$ we have

$$
\begin{aligned}
& \limsup _{k \rightarrow \infty}\left(\left(\left.\left(\left.\mathcal{L}_{\varphi \circ \pi_{1}}^{n}\right|_{V}\right)^{*} \mathcal{L}_{\varphi \circ \pi_{1}}^{n}\right|_{V}\right)^{k}\left(\mathbb{1}_{\left\{\pi_{2}=g\right\}}\right), \mathbb{1}_{\left\{\pi_{2}=g^{\prime}\right\}}\right)^{1 / k} \\
\leq & c_{n}\left(N_{n}+1\right) \max \left\{\mathrm{e}^{\left(2 n+N_{n}\right) \mathcal{P}\left(\varphi, \Psi^{-1}\left\{g^{-1} g^{\prime}\right\} \cap \Sigma^{*}\right)}, \mathrm{e}^{2 n \mathcal{P}\left(\varphi, \Psi^{-1}\left\{g^{-1} g^{\prime}\right\} \cap \Sigma^{*}\right)}\right\} .
\end{aligned}
$$

Combining (3.6), (3.7) and (3.8), we obtain that

$$
\begin{aligned}
& \rho\left(\left.\mathcal{L}_{\varphi \circ \pi_{1}}\right|_{V}\right)=\lim _{n \rightarrow \infty}\left\|\left.\left(\left.\mathcal{L}_{\varphi \circ \pi_{1}}^{n}\right|_{V}\right)^{*} \mathcal{L}_{\varphi \circ \pi_{1}}^{n}\right|_{V}\right\|^{1 /(2 n)} \\
& \leq \limsup _{n \rightarrow \infty}\left(c _ { n } ( N _ { n } + 1 ) \operatorname { s u p } _ { g , g ^ { \prime } \in G } \operatorname { m a x } \left\{\mathrm{e}^{\left(2 n+N_{n}\right) \mathcal{P}\left(\varphi, \Psi^{-1}\left\{g^{-1} g^{\prime}\right\} \cap \Sigma^{*}\right),}\right.\right. \\
& \left.\left.\mathrm{e}^{2 n \mathcal{P}\left(\varphi, \Psi^{-1}\left\{g^{-1} g^{\prime}\right\} \cap \Sigma^{*}\right)}\right\}\right)^{1 /(2 n)} \\
& \leq \lim _{n \rightarrow \infty}\left(c_{n}\left(N_{n}+1\right)\right)^{1 /(2 n)} \sup _{g \in G} \max \left\{\mathrm{e}^{\left(1+N_{n} /(2 n)\right) \mathcal{P}\left(\varphi, \Psi^{-1}\{g\} \cap \Sigma^{*}\right)}, \mathrm{e}^{\mathcal{P}\left(\varphi, \Psi^{-1}\{g\} \cap \Sigma^{*}\right)}\right\} .
\end{aligned}
$$

Since $\lim _{n \rightarrow \infty}\left(c_{n}\right)^{1 / n}=1$ and $\lim _{n \rightarrow \infty} n^{-1} N_{n}=0$, the proof is complete.

In the following definition, we introduce certain important closed linear subspaces of the space $L^{2}\left(\Sigma \times G, \mu_{\varphi} \times \lambda\right)$.

Definition 3.12. For $j \in \mathbb{N}_{0}$, let $V_{j} \subset L^{2}\left(\Sigma \times G, \mu_{\varphi} \times \lambda\right)$ denote the subspace consisting of all $f \in L^{2}\left(\Sigma \times G, \mu_{\varphi} \times \lambda\right)$ which possess a $\mathcal{C}(j) \otimes \mathcal{B}(G)$-measurable representative in $L^{2}\left(\Sigma \times G, \mu_{\varphi} \times \lambda\right)$, where $\mathcal{C}(j) \otimes \mathcal{B}(G)$ denotes the product $\sigma$ algebra of $\mathcal{C}(j)$ and the Borel $\sigma$-algebra $\mathcal{B}(G)$ on $G$.

Note that $V_{j}$ is a Hilbert space for each $j \in \mathbb{N}_{0}$. The next lemma gives an invariance property for $V_{j}$ with respect to $\mathcal{L}_{\varphi \circ \pi_{1}}$ for $\mathcal{C}(k)$-measurable potentials $\varphi$. 
Lemma 3.13. Let $\varphi: \Sigma \rightarrow \mathbb{R}$ be $\mathcal{C}(k)$-measurable for some $k \in \mathbb{N}_{0}$. Then $V_{j}$ is $\mathcal{L}_{\varphi \circ \pi_{1}}$-invariant for each $j \in \mathbb{N}$ with $j \geq k-1$. Moreover, for all $j \in \mathbb{N}_{0}$ we have that $U\left(V_{j}\right) \subset V_{j+1}$.

Proof. If $f$ is $\mathcal{C}(j)$-measurable, $j \in \mathbb{N}_{0}$, then it follows from Lemma 3.5 (2) that $\mathcal{L}_{\varphi \circ \pi_{1}}(f)$ is given by

$$
\mathcal{L}_{\varphi \circ \pi_{1}}(f)(\omega, g)=\sum_{i \in I: i \omega_{1} \in \Sigma^{2}} \mathrm{e}^{\varphi(i \omega)} f\left(i \omega, g \Psi(i)^{-1}\right) .
$$

Note that the right-hand side of the previous equation depends only on $g \in G$ and on the elements $\omega_{1}, \ldots, \omega_{\max \{k-1, j-1,1\}} \in I$. Consequently, for $j \in \mathbb{N}$ with $j \geq k-1$, we have that $V_{j}$ is $\mathcal{L}_{\varphi \circ \pi_{1}}$-invariant.

The remaining assertion follows immediately from the definition of $U$.

We need the following notion of symmetry.

Definition 3.14. We say that $\varphi: \Sigma \rightarrow \mathbb{R}$ is asymptotically symmetric with respect to $\Psi$ if there exist sequences $\left(c_{m}\right)_{m \in \mathbb{N}} \in\left(\mathbb{R}^{+}\right)^{\mathbb{N}}$ and $\left(N_{m}\right)_{m \in \mathbb{N}} \in \mathbb{N}_{0}^{\mathbb{N}}$, satisfying $\lim _{m}\left(c_{m}\right)^{1 / m}=1$ and $\lim _{m} m^{-1} N_{m}=0$, such that for each $g \in G$ and for all $n \in \mathbb{N}$, we have

$$
\sum_{\omega \in \Sigma^{n}: \Psi(\omega)=g} \mathrm{e}^{S_{\omega} \varphi} \leq c_{n} \sum_{i=0}^{N_{n}} \sum_{\tau \in \Sigma^{n+i}: \Psi(\tau)=g^{-1}} \mathrm{e}^{S_{\tau} \varphi} .
$$

Remark 3.15. If $\varphi$ is asymptotically symmetric with respect to $\Psi$, then it is straightforward to verify that, for each $\psi: \Sigma \rightarrow \mathbb{R}^{+}$Hölder continuous and $c \in \mathbb{R}$, we have that also $\varphi+\log \psi-\log \psi \circ \sigma+c$ is asymptotically symmetric with respect to $\Psi$. Using the Gibbs property (2.1) of $\mu_{\varphi}$, an equivalent way to state that $\varphi$ is asymptotically symmetric with respect to $\Psi$ is the following: there exist sequences $\left(c_{m}^{\prime}\right)_{m \in \mathbb{N}} \in\left(\mathbb{R}^{+}\right)^{\mathbb{N}}$ and $\left(N_{m}^{\prime}\right)_{m \in \mathbb{N}} \in \mathbb{N}_{0}^{\mathbb{N}}$, satisfying $\lim _{m}\left(c_{m}^{\prime}\right)^{1 / m}=1$ and $\lim _{m} m^{-1} N_{m}^{\prime}=0$, such that for each $g \in G$ and for all $n \in \mathbb{N}$, we have

$$
\sum_{\omega \in \Sigma^{n}: \Psi(\omega)=g} \mu_{\varphi}([\omega]) \leq c_{n}^{\prime} \sum_{i=0}^{N_{n}^{\prime}} \sum_{\tau \in \Sigma^{n+i}: \Psi(\tau)=g^{-1}} \mu_{\varphi}([\tau]) .
$$

The next lemma gives a necessary and sufficient condition for $\left.\mathcal{L}_{\varphi \circ \pi_{1}}\right|_{V_{j}}$ to be asymptotically self-adjoint.

Lemma 3.16. Let $\varphi: \Sigma \rightarrow \mathbb{R}$ be $\mathcal{C}(k)$-measurable, for some $k \in \mathbb{N}_{0}$. For each $j \in \mathbb{N}$ with $j \geq k-1$, we then have that $\varphi$ is asymptotically symmetric with respect to $\Psi$ if and only if $\left.\mathcal{L}_{\varphi \circ \pi_{1}}\right|_{V_{j}}$ is asymptotically self-adjoint.

Proof. We first observe that by Lemma 3.6 and by the Gibbs property (2.1) of $\mu_{\varphi}$, there exists $K>0$ such that for all $n \in \mathbb{N}$ and for all $g, g^{\prime} \in G$ we have

$$
K^{-1} \leq \frac{\left(\mathcal{L}_{\varphi \circ \pi_{1}}^{n}\left(\mathbb{1}_{\Sigma \times\{g\}}\right), \mathbb{1}_{\Sigma \times\left\{g^{\prime}\right\}}\right)}{\sum_{\tau \in \Sigma^{n}: g \Psi(\tau)=g^{\prime}} \mathrm{e}^{S_{\tau} \varphi}} \leq K
$$

unless the nominator and denominator in (3.10) are both equal to zero. From (3.10) we obtain that $\varphi$ is asymptotically symmetric with respect to $\Psi$ if and only if there 
exist sequences $\left(c_{m}\right) \in\left(\mathbb{R}^{+}\right)^{\mathbb{N}}$ and $\left(N_{m}\right) \in \mathbb{N}_{0}^{\mathbb{N}}$, as in Definition 3.14 such that for all $n \in \mathbb{N}$ and $g, g^{\prime} \in G$ we have

$$
\left(\mathcal{L}_{\varphi \circ \pi_{1}}^{n}\left(\mathbb{1}_{\Sigma \times\{g\}}\right), \mathbb{1}_{\Sigma \times\left\{g^{\prime}\right\}}\right) \leq c_{n}\left(\mathbb{1}_{\Sigma \times\{g\}}, \sum_{i=0}^{N_{n}} \mathcal{L}_{\varphi \circ \pi_{1}}^{n+i}\left(\mathbb{1}_{\Sigma \times\left\{g^{\prime}\right\}}\right)\right) .
$$

Since $V_{0} \subset V_{j}$ for each $j \in \mathbb{N}_{0}$, we obtain that if $\left.\mathcal{L}_{\varphi \circ \pi_{1}}\right|_{V_{j}}$ is asymptotically selfadjoint, then $\varphi$ is asymptotically symmetric with respect to $\Psi$.

For the opposite implication, let $j \in \mathbb{N}, j \geq k-1$ and suppose that $\varphi$ is asymptotically symmetric with respect to $\Psi$. By Lemma 3.13, we have that $V_{j}$ is $\mathcal{L}_{\varphi \circ \pi_{1}}$ invariant. Next, we note that since $\varphi$ is asymptotically symmetric with respect to $\Psi$, we have that, for each $\omega \in \Sigma^{j}$, there exists $\kappa(\omega) \in \Sigma^{*}$ such that $\Psi(\omega) \Psi(\kappa(\omega))=$ id. Combining this with item (3) of our standing assumptions, we conclude that for all $\omega, \omega^{\prime} \in \Sigma^{j}$ there exists a finite-to-one map which maps $\tau \in \Sigma^{*}$ to an element $\omega \gamma_{1} \kappa(\omega) \gamma_{2} \tau \gamma_{3} \omega^{\prime} \in \Sigma^{*}$, where $\Psi\left(\gamma_{i}\right)=$ id and $\gamma_{i}$ depends only on the preceding and successive symbol, for each $i \in\{1,2,3\}$. Hence, in view of Lemma 3.6 and the Gibbs property (2.1) of $\mu_{\varphi}$, and by using that $\Sigma^{j}$ is finite, we conclude that there exist $N \in \mathbb{N}$ and $C>0$ (depending on $j$ ) such that for all $n \in \mathbb{N}, g, g^{\prime} \in G$ and for all $\omega, \omega^{\prime} \in \Sigma^{j}$ we have

$$
\left(\mathcal{L}_{\varphi \circ \pi_{1}}^{n}\left(\mathbb{1}_{\Sigma \times\{g\}}\right), \mathbb{1}_{\Sigma \times\left\{g^{\prime}\right\}}\right) \leq C \sum_{r=0}^{N}\left(\mathcal{L}_{\varphi \circ \pi_{1}}^{n+r}\left(\mathbb{1}_{[\omega] \times\{g\}}\right), \mathbb{1}_{\left[\omega^{\prime}\right] \times\left\{g^{\prime}\right\}}\right) .
$$

By first using (3.11) and then (3.12), we deduce that for all $n \in \mathbb{N}, g, g^{\prime} \in G$ and for all $\omega, \omega^{\prime} \in \Sigma^{j}$,

$$
\begin{aligned}
\left(\mathcal{L}_{\varphi \circ \pi_{1}}^{n}\left(\mathbb{1}_{\Sigma \times\{g\}}\right), \mathbb{1}_{\Sigma \times\left\{g^{\prime}\right\}}\right) & \leq c_{n}\left(\mathbb{1}_{\Sigma \times\{g\}}, \sum_{i=0}^{N_{n}} \mathcal{L}_{\varphi \circ \pi_{1}}^{n+i}\left(\mathbb{1}_{\Sigma \times\left\{g^{\prime}\right\}}\right)\right) \\
& \leq c_{n} C \sum_{i=0}^{N_{n}} \sum_{r=0}^{N}\left(\mathbb{1}_{[\omega] \times\{g\}}, \mathcal{L}_{\varphi \circ \pi_{1}}^{n+i+r}\left(\mathbb{1}_{\left[\omega^{\prime}\right] \times\left\{g^{\prime}\right\}}\right)\right) \\
& \leq c_{n} C N \sum_{i=0}^{N_{n}+N}\left(\mathbb{1}_{[\omega] \times\{g\}}, \mathcal{L}_{\varphi \circ \pi_{1}}^{n+i}\left(\mathbb{1}_{\left[\omega^{\prime}\right] \times\left\{g^{\prime}\right\}}\right)\right) .
\end{aligned}
$$

Since $\left(\mathcal{L}_{\varphi \circ \pi_{1}}^{n}\left(\mathbb{1}_{[\omega] \times\{g\}}\right), \mathbb{1}_{\left[\omega^{\prime}\right] \times\left\{g^{\prime}\right\}}\right) \leq\left(\mathcal{L}_{\varphi \circ \pi_{1}}^{n}\left(\mathbb{1}_{\Sigma \times\{g\}}\right), \mathbb{1}_{\Sigma \times\left\{g^{\prime}\right\}}\right)$ for all $\omega, \omega^{\prime} \in \Sigma^{j}$, it follows that $\left.\mathcal{L}_{\varphi \circ \pi_{1}}\right|_{V_{j}}$ is asymptotically self-adjoint with respect to the sequences $\left(c_{m}^{\prime}\right) \in\left(\mathbb{R}^{+}\right)^{\mathbb{N}}$ and $\left(N_{m}^{\prime}\right) \in \mathbb{N}_{0}^{\mathbb{N}}$, which are given by $c_{m}^{\prime}:=c_{m} C N$ and $N_{m}^{\prime}:=N_{m}+N$. The proof is complete.

The following corollary is a consequence of Proposition 3.11 and clarifies the relation between $\sup _{g \in G}\left\{\mathcal{P}\left(\varphi, \Psi^{-1}\{g\} \cap \Sigma^{*}\right)\right\}$ and the spectral radius of $\left.\mathcal{L}_{\varphi \circ \pi_{1}}\right|_{V_{j}}$ provided that $\varphi$ is asymptotically symmetric with respect to $\Psi$.

Corollary 3.17. Let $\varphi: \Sigma \rightarrow \mathbb{R}$ be $\mathcal{C}(k)$-measurable, for some $k \in \mathbb{N}_{0}$, and suppose that $\varphi$ is asymptotically symmetric with respect to $\Psi$. For each $j \in \mathbb{N}$ with $j \geq k-1$, we then have that

$$
\sup _{g \in G}\left\{\mathcal{P}\left(\varphi, \Psi^{-1}\{g\} \cap \Sigma^{*}\right)\right\}=\log \rho\left(\left.\mathcal{L}_{\varphi \circ \pi_{1}}\right|_{V_{j}}\right) .
$$


Proof. Fix $j \in \mathbb{N}$ with $j \geq k-1$. By Lemma 3.13, we then have that $V_{j}$ is $\mathcal{L}_{\varphi \circ \pi_{1}}$ invariant. Let us first verify that without loss of generality we may assume that $\mathcal{L}_{\varphi} \mathbb{1}=\mathbb{1}$. Otherwise, by Theorem 2.6, there exists a $\mathcal{C}(\max \{k-1,1\})$-measurable function $h: \Sigma \rightarrow \mathbb{R}^{+}$with $\mathcal{L}_{\varphi}(h)=\mathrm{e}^{\mathcal{P}(\varphi)} h$. For $\tilde{\varphi}:=\varphi+\log h-\log h \circ \sigma-\mathcal{P}(\varphi)$, we then have that $\mathcal{L}_{\tilde{\varphi}} \mathbb{1}=\mathbb{1}, \mathcal{P}(\tilde{\varphi})=0$ and, for each $g \in G$,

$$
\mathcal{P}\left(\tilde{\varphi}, \Psi^{-1}\{g\} \cap \Sigma^{*}\right)=\mathcal{P}\left(\varphi, \Psi^{-1}\{g\} \cap \Sigma^{*}\right)-\mathcal{P}(\varphi) .
$$

Further, we have that $\tilde{\varphi}$ is asymptotically symmetric with respect to $\Psi$, by Remark 3.15. It remains to show that $V_{j}$ is $\mathcal{L}_{\tilde{\varphi} \circ \pi_{1}}$-invariant and that

$$
\log \rho\left(\left.\mathcal{L}_{\tilde{\varphi} \circ \pi_{1}}\right|_{V_{j}}\right)=\log \rho\left(\left.\mathcal{L}_{\varphi \circ \pi_{1}}\right|_{V_{j}}\right)-\mathcal{P}(\varphi) .
$$

Since $h$ is $\mathcal{C}(\max \{k-1,1\})$-measurable, we have that $V_{j}$ is $M_{h \circ \pi_{1}}$-invariant and, by the definition of the Perron-Frobenius operator, we obtain that

$$
\left.\mathcal{L}_{\tilde{\varphi} \circ \pi_{1}}\right|_{V_{j}}=\mathrm{e}^{-\mathcal{P}(\varphi)}\left(\left.M_{h \circ \pi_{1}}\right|_{V_{j}}\right)^{-1} \circ\left(\left.\mathcal{L}_{\varphi \circ \pi_{1}}\right|_{V_{j}}\right) \circ\left(\left.M_{h \circ \pi_{1}}\right|_{V_{j}}\right) .
$$

We conclude that $V_{j}$ is $\mathcal{L}_{\tilde{\varphi} \circ \pi_{1}}$-invariant and that $\left.\mathcal{L}_{\tilde{\varphi} \circ \pi_{1}}\right|_{V_{j}}$ and $\left.\mathrm{e}^{-\mathcal{P}(\varphi)} \mathcal{L}_{\varphi \circ \pi_{1}}\right|_{V_{j}}$ have the same spectrum. The latter fact gives the equality in (3.13). Hence, we may assume without loss of generality that $\mathcal{L}_{\varphi} \mathbb{1}=\mathbb{1}$.

By Lemma 3.16. we have that $\left.\mathcal{L}_{\varphi \circ \pi_{1}}\right|_{V_{j}}$ is asymptotically self-adjoint. Since $V_{j}$ is a closed linear subspace of $L^{2}\left(\Sigma \times G, \mu_{\varphi} \times \lambda\right)$ such that $\left\{f^{-}: f \in V_{j}\right\} \cup$ $\left\{\mathbb{1}_{\left\{\pi_{2}=g\right\}}: g \in G\right\} \subset V_{j}$, the assertion of the corollary follows from Proposition 3.11 .

Remark. Note that, in particular, under the assumptions of the previous corollary we have that $\rho\left(\left.\mathcal{L}_{\varphi \circ \pi_{1}}\right|_{V_{j}}\right)$ is independent of $j \in \mathbb{N}$ for all $j \geq k-1$.

3.2. Random walks on graphs and amenability. In this section we relate the Perron-Frobenius operator to the transition operator of a certain random walk on a graph. We start by introducing the following graphs.

Definition 3.18. For each $j \in \mathbb{N}_{0}$, the $j$-step graph of $(\Sigma \times G, \sigma \rtimes \Psi)$ consists of the vertex set $\Sigma^{j} \times G$ where two vertices $(\omega, g),\left(\omega^{\prime}, g^{\prime}\right) \in \Sigma^{j} \times G$ are connected by an edge in $X_{j}$ if and only if

$$
(\sigma \rtimes \Psi)^{-1}([\omega] \times\{g\}) \cap\left(\left[\omega^{\prime}\right] \times\left\{g^{\prime}\right\}\right) \neq \varnothing
$$

or

$$
(\sigma \rtimes \Psi)^{-1}\left(\left[\omega^{\prime}\right] \times\left\{g^{\prime}\right\}\right) \cap([\omega] \times\{g\}) \neq \varnothing .
$$

We use $X_{j}(\Sigma \times G, \sigma \rtimes \Psi)$ or simply $X_{j}$ to denote this graph.

Provided that $\Psi\left(\Sigma^{*}\right)=G$, we have that each $j$-step graph of $(\Sigma \times G, \sigma \rtimes \Psi)$ is connected. The next lemma shows that each of these graphs is roughly isometric to the Cayley graph of $G$ with respect to $\Psi(I) \cup \Psi(I)^{-1}$ denoted by $X(G, \Psi(I) \cup$ $\left.\Psi(I)^{-1}\right)$. For a similar argument, see OW07.

Lemma 3.19. Suppose that $\Psi\left(\Sigma^{*}\right)=G$ and let $j \in \mathbb{N}_{0}$. We then have that the graphs $X_{j}(\Sigma \times G, \sigma \rtimes \Psi)$ and $X\left(G, \Psi(I) \cup \Psi(I)^{-1}\right)$ are roughly isometric. 
Proof. By identifying $\Sigma^{0} \times G$ with $G$, we have that $X_{0}$ is isometric to $X(G, \Psi(I) \cup$ $\left.\Psi(I)^{-1}\right)$. Suppose now that $j \in \mathbb{N}$. We show that the map $\pi_{2}: \Sigma^{j} \times G \rightarrow G$, given by $\pi_{2}(\omega, g):=g$, for all $(\omega, g) \in \Sigma^{j} \times G$, defines a rough isometry between the metric spaces $\left(\Sigma^{j} \times G, d_{j}\right)$ and $(G, d)$, where $d_{j}$ denotes the graph metric on $X_{j}$ and $d$ denotes the graph metric on $X\left(G, \Psi(I) \cup \Psi(I)^{-1}\right)$. Clearly, we have that $\pi_{2}$ is surjective. Further, by the definition of the edge set of $X_{j}$, we have that if two vertices $(\omega, g),\left(\omega^{\prime}, g^{\prime}\right) \in \Sigma^{j} \times G$ are connected by an edge in $X_{j}$, then $g$ and $g^{\prime}$ are connected by an edge in $X\left(G, \Psi(I) \cup \Psi(I)^{-1}\right)$. Hence, for all $(\omega, g),\left(\omega^{\prime}, g^{\prime}\right) \in$ $\Sigma^{j} \times G$ we have that $d\left(\pi_{2}(\omega, g), \pi_{2}\left(\omega^{\prime}, g^{\prime}\right)\right) \leq d_{j}\left((\omega, g),\left(\omega^{\prime}, g^{\prime}\right)\right)$.

It remains to show that there exist constants $A, B>0$ such that for all $(\omega, g)$, $\left(\omega^{\prime}, g^{\prime}\right) \in \Sigma^{j} \times G$

$$
d_{j}\left((\omega, g),\left(\omega^{\prime}, g^{\prime}\right)\right) \leq A d\left(\pi_{2}(\omega, g), \pi_{2}\left(\omega^{\prime}, g^{\prime}\right)\right)+B .
$$

First note that by our assumptions, there exists a finite set $F \subset \Sigma^{*}$ with the following properties.

(1) For all $\tau \in \Sigma^{j}$ there exists $\kappa(\tau) \in F$ such that $\Psi(\tau) \Psi(\kappa(\tau))=$ id, and for all $h \in \Psi(I) \cup \Psi(I)^{-1}$ there is $\alpha \in F$ such that $\Psi(\alpha)=h$. (We used that card $(I)<\infty$, and hence card $\left(\Sigma^{j}\right)<\infty$, and that $\Psi\left(\Sigma^{*}\right)=G$.)

(2) For all $a, b \in I$ there exists $\gamma \in F \cap \Psi^{-1}\{\mathrm{id}\} \cup\{\varnothing\}$ such that $a \gamma b \in \Sigma^{*}$. (We used card $(I)<\infty$ and item (3) of our standing assumptions.)

Setting $L:=\max _{\gamma \in F}|\gamma|, A:=2 L$ and $B:=3 L+j$, we will show that (3.14) holds. Let $(\omega, g),\left(\omega^{\prime}, g^{\prime}\right) \in \Sigma^{j} \times G$ be given. First suppose that $d\left(\pi_{2}(\omega, g), \pi_{2}\left(\omega^{\prime}, g^{\prime}\right)\right)=$ $m \in \mathbb{N}$. Hence, there exist $h_{1}, \ldots, h_{m} \in \Psi(I) \cup \Psi(I)^{-1}$ such that $g h_{1} \ldots h_{m}=g^{\prime}$. By property (1) above, there exist $\alpha_{1}, \ldots, \alpha_{m} \in F$ such that $\Psi\left(\alpha_{i}\right)=h_{i}$ for all $1 \leq i \leq m$, and there exists $\kappa(\omega) \in F$ such that $\Psi(\omega) \Psi(\kappa(\omega))=$ id. Then property (2) implies the existence of $\gamma_{0}, \gamma_{1}, \ldots, \gamma_{m+1} \in F \cap \Psi^{-1}\{\mathrm{id}\} \cup\{\varnothing\}$ such that $\omega \gamma_{0} \kappa(\omega) \gamma_{1} \alpha_{1} \gamma_{2} \alpha_{2} \ldots \gamma_{m} \alpha_{m} \gamma_{m+1} \omega^{\prime} \in \Sigma^{*}$, and hence

$$
\left[\omega \gamma_{0} \kappa(\omega) \gamma_{1} \alpha_{1} \gamma_{2} \alpha_{2} \ldots \gamma_{m} \alpha_{m} \gamma_{m+1} \omega^{\prime}\right] \subset([\omega] \times\{g\}) \cap(\sigma \rtimes \Psi)^{-l}\left(\left[\omega^{\prime}\right] \times\left\{g^{\prime}\right\}\right),
$$

where we have set $l:=\left|\omega \gamma_{0} \kappa(\omega) \gamma_{1} \alpha_{1} \gamma_{2} \alpha_{2} \ldots \gamma_{m} \alpha_{m} \gamma_{m+1}\right| \leq(2 m+3) L+j$. The inequality in (3.14) follows. Finally, if $d\left(\pi_{2}(\omega, g), \pi_{2}\left(\omega^{\prime}, g^{\prime}\right)\right)=0$, then $g=g^{\prime}$ and there exist $\gamma_{0}, \gamma_{1} \in F \cap \Psi^{-1}\{$ id $\} \cup\{\varnothing\}$ such that $\omega \gamma_{0} \kappa(\omega) \gamma_{1} \omega^{\prime} \in \Sigma^{*}$. Hence, $d_{j}\left((\omega, g),\left(\omega^{\prime}, g^{\prime}\right)\right) \leq B$. The proof is complete.

In the following proposition we let $\mathbb{E}(\cdot \mid \mathcal{C}(j)): L^{2}\left(\Sigma \times G, \mu_{\varphi} \times \lambda\right) \rightarrow V_{j}$ denote the conditional expectation given $\mathcal{C}(j)$.

Proposition 3.20. Suppose that $\Psi\left(\Sigma^{*}\right)=G$. Let $\varphi: \Sigma \rightarrow \mathbb{R}$ be $\mathcal{C}(k)$-measurable for some $k \in \mathbb{N}_{0}$, such that $\mathcal{L}_{\varphi} \mathbb{1}=\mathbb{1}$. The following holds for all $j \in \mathbb{N}$ with $j \geq k-1$. For the bounded linear operator $\mathbb{E}(U(\cdot) \mid \mathcal{C}(j)): V_{j} \rightarrow V_{j}$ we have that

$$
\rho(\mathbb{E}(U(\cdot) \mid \mathcal{C}(j))) \leq\|\mathbb{E}(U(\cdot) \mid \mathcal{C}(j))\|=1
$$

with equality if and only if $G$ is amenable. In particular, we have that

$$
\rho\left(\left.\mathcal{L}_{\varphi \circ \pi_{1}}\right|_{V_{j}}\right) \leq\left\|\left.\mathcal{L}_{\varphi \circ \pi_{1}}\right|_{V_{j}}\right\|=1
$$

with equality if and only if $G$ is amenable. 
Proof. Fix $j \in \mathbb{N}$ with $j \geq k-1$. We first observe that for each $f \in V_{j}$ we have that $\mathbb{E}(U(f) \mid \mathcal{C}(j))$ is the unique element in $V_{j}$, such that $(\mathbb{E}(U(f) \mid \mathcal{C}(j)), g)=$ $(U(f), g)$ for all $g \in V_{j}$. Since $(U(f), g)=\left(f, \mathcal{L}_{\varphi \circ \pi_{1}}(g)\right)$ and $V_{j}$ is $\mathcal{L}_{\varphi \circ \pi_{1}}$-invariant by Lemma 3.13, we conclude that $\mathbb{E}(U(\cdot) \mid \mathcal{C}(j))$ is the adjoint of $\left.\mathcal{L}_{\varphi \circ \pi_{1}}\right|_{V_{j}}$. Since $U\left(V_{0}\right) \subset V_{1} \subset V_{j}$, we have that the restriction of $\mathbb{E}(U(\cdot) \mid \mathcal{C}(j))$ to $V_{0}$ is equal to $\left.U\right|_{V_{0}}$. Because $U$ is an isometry by Lemma 3.5 (1), we conclude that $\left\|\left.\mathcal{L}_{\varphi \circ \pi_{1}}\right|_{V_{j}}\right\|=$ $\|\mathbb{E}(U(\cdot) \mid \mathcal{C}(j))\|=1$.

In order to prove the amenability dichotomy for $\rho(\mathbb{E}(U(\cdot) \mid \mathcal{C}(j)))$ we aim to apply Theorem 2.20 to a transition matrix on the vertex set $\Sigma^{j} \times G$ of the graph $X_{j}$. Since a basis of $V_{j}$ is given by $\left\{\mathbb{1}_{[\omega] \times\{g\}}:(\omega, g) \in \Sigma^{j} \times G\right\}$, we obtain a Hilbert space isomorphism between $V_{j}$ and $\ell^{2}\left(\Sigma^{j} \times G, \nu_{j}\right)$ by setting $\nu_{j}(\omega, g):=$ $\left(\mu_{\varphi} \times \lambda\right)([\omega] \times\{g\})$ for every $(\omega, g) \in \Sigma^{j} \times G$. Using this isomorphism and with respect to the canonical basis of $\ell^{2}\left(\Sigma^{j} \times G, \nu_{j}\right)$, we have that $\mathbb{E}(U(\cdot) \mid \mathcal{C}(j))$ is represented by the matrix $P=\left(p\left((\omega, g),\left(\omega^{\prime}, g^{\prime}\right)\right)\right)$ given by

$$
p\left((\omega, g),\left(\omega^{\prime}, g^{\prime}\right)\right)=\left(U \mathbb{1}_{\left[\omega^{\prime}\right] \times\left\{g^{\prime}\right\}}, \mathbb{1}_{[\omega] \times\{g\}}\right)\left(\left(\mu_{\varphi} \times \lambda\right)([\omega] \times\{g\})\right)^{-1} .
$$

Note that we have chosen the matrix $P$ to act on the left. Summing over $\left(\omega^{\prime}, g^{\prime}\right) \in$ $\Sigma^{j} \times G$ in the previous line, we obtain that $P$ is a transition matrix on $\Sigma^{j} \times G$. Using that $\mu_{\varphi} \times \lambda$ is $(\sigma \rtimes \Psi)$-invariant by Lemma 3.3, one then deduces from (3.15) that $\nu_{j}$ is $P$-invariant. Let us now verify that Theorem 2.20 is applicable to the transition matrix $P$ acting on the vertex set $\Sigma^{j} \times G$ of $X_{j}$. Since card $(I)<\infty$, we have that $X_{j}$ has bounded geometry. Further, it follows immediately from the definition of $X_{j}$ that $p\left((\omega, g),\left(\omega^{\prime}, g^{\prime}\right)\right)>0$ implies that $(\omega, g) \sim\left(\omega^{\prime}, g^{\prime}\right)$ in $X_{j}$, and hence $P$ has bounded range $(R=1)$ with respect to $X_{j}$. It is also clear from the definition of $\nu_{j}$ that

$0<\min _{\omega \in \Sigma^{j}} \mu_{\varphi}([\omega])=\inf _{(\omega, g) \in \Sigma^{j} \times G} \nu_{j}(\omega, g) \leq \sup _{(\omega, g) \in \Sigma^{j} \times G} \nu_{j}(\omega, g)=\max _{\omega \in \Sigma^{j}} \mu_{\varphi}([\omega])<\infty$.

It remains to verify that $P$ is uniformly irreducible with respect to $X_{j}$. Let $(\omega, g),\left(\omega^{\prime}, g^{\prime}\right) \in \Sigma^{j} \times G$ denote a pair of vertices which is connected by an edge in $X_{j}$. By definition, we then have that $(\sigma \rtimes \Psi)^{-1}\left(\left[\omega^{\prime}\right] \times\left\{g^{\prime}\right\}\right) \cap([\omega] \times\{g\}) \neq$ $\varnothing$ or $(\sigma \rtimes \Psi)^{-1}([\omega] \times\{g\}) \cap\left(\left[\omega^{\prime}\right] \times\left\{g^{\prime}\right\}\right) \neq \varnothing$. In the first case, we have that

$$
\begin{aligned}
p\left((\omega, g),\left(\omega^{\prime}, g^{\prime}\right)\right) & =\left(\mu_{\varphi} \times \lambda\right)\left((\sigma \rtimes \Psi)^{-1}\left(\left[\omega^{\prime}\right] \times\left\{g^{\prime}\right\}\right) \cap([\omega] \times\{g\})\right)\left(\mu_{\varphi}([\omega])\right)^{-1} \\
& =\mu_{\varphi}\left(\left[\omega \omega_{j}^{\prime}\right]\right)\left(\mu_{\varphi}([\omega])\right)^{-1} \geq \min _{\tau \in \Sigma^{j+1}} \mu_{\varphi}([\tau])>0 .
\end{aligned}
$$

Next we consider the second case in which $(\sigma \rtimes \Psi)^{-1}([\omega] \times\{g\}) \cap\left(\left[\omega^{\prime}\right] \times\left\{g^{\prime}\right\}\right) \neq \varnothing$, and thus $g^{\prime} \Psi\left(\omega_{1}^{\prime}\right)=g$. Similarly as in the proof of Lemma 3.19 one can verify that there exists a finite set $F \subset \Sigma^{*}$ with the following properties. First, for all $\tau \in \Sigma^{j} \cup I$ there exists $\kappa(\tau) \in F$ such that $\Psi(\tau) \Psi(\kappa(\tau))=$ id, and second, for all $a, b \in I$ there exists $\gamma \in F \cap \Psi^{-1}\{\mathrm{id}\} \cup\{\varnothing\}$ such that $a \gamma b \in \Sigma^{*}$. Hence, there exist $\gamma_{1}, \gamma_{2}, \gamma_{3} \in F$ such that

$$
\left(\left[\omega \gamma_{1} \kappa(\omega) \gamma_{2} \kappa\left(\omega_{1}^{\prime}\right) \gamma_{3} \omega^{\prime}\right] \times\{g\}\right) \subset([\omega] \times\{g\}) \cap(\sigma \rtimes \Psi)^{-l}\left(\left[\omega^{\prime}\right] \times\left\{g^{\prime}\right\}\right),
$$

where we have set $l:=\left|\omega \gamma_{1} \kappa(\omega) \gamma_{2} \kappa\left(\omega_{1}^{\prime}\right) \gamma_{3}\right| \leq j+5 \max _{\gamma \in F}|\gamma|$. Consequently,

$$
p^{(l)}\left((\omega, g),\left(\omega^{\prime}, g^{\prime}\right)\right) \geq\left(\min _{\tau \in \Sigma^{j+1}} \mu_{\varphi}([\tau])\right)^{j+5 \max _{\gamma \in F}|\gamma|}>0 .
$$


Hence, with $K:=j+5 \max _{\gamma \in F}|\gamma|$ and $\epsilon:=\left(\min _{\tau \in \Sigma^{j+1}} \mu_{\varphi}([\tau])\right)^{j+5 \max _{\gamma \in F}|\gamma|}>0$ we have that $P$ is uniformly irreducible with respect to $X_{j}$.

We are now in the position to apply Theorem 2.20 to the transition matrix $P$, which gives that $\rho(P)=1$ if and only if $X_{j}$ is amenable. Since $X_{j}$ is roughly isometric to the Cayley graph of $G$ with respect to $\Psi(I) \cup \Psi(I)^{-1}$ by Lemma 3.19, it follows from Theorem 2.15 that $X_{j}$ is amenable if and only if $G$ is amenable (cf. Proposition 2.17) . Finally, since $\mathbb{E}(U(\cdot) \mid \mathcal{C}(j))$ and $P$ are conjugated by an isomorphism of Hilbert spaces, we have $\rho(\mathbb{E}(U(\cdot) \mid \mathcal{C}(j)))=\rho(P)$, which completes the proof.

Summarizing the outcomes of this section, we obtain the following main result.

Theorem 3.21. Suppose that $\Psi\left(\Sigma^{*}\right)=G$ and let $\varphi: \Sigma \rightarrow \mathbb{R}$ be $\mathcal{C}(k)$-measurable for some $k \in \mathbb{N}_{0}$. The following holds for all $j \in \mathbb{N}$ with $j \geq k-1$. We have

$$
\mathcal{P}\left(\varphi, \Psi^{-1}\{\operatorname{id}\} \cap \Sigma^{*}\right) \leq \log \rho\left(\left.\mathcal{L}_{\varphi \circ \pi_{1}}\right|_{V_{j}}\right) \leq \log \rho\left(\mathcal{L}_{\varphi \circ \pi_{1}}\right)=\mathcal{P}(\varphi),
$$

with equality in the second inequality if and only if $G$ is amenable. Moreover, if $\varphi$ is asymptotically symmetric with respect to $\Psi$, then

$$
\mathcal{P}\left(\varphi, \Psi^{-1}\{\mathrm{id}\} \cap \Sigma^{*}\right)=\log \rho\left(\left.\mathcal{L}_{\varphi \circ \pi_{1}}\right|_{V_{j}}\right),
$$

and so $G$ is amenable if and only if $\mathcal{P}\left(\varphi, \Psi^{-1}\{\operatorname{id}\} \cap \Sigma^{*}\right)=\mathcal{P}(\varphi)$.

Proof. Fix $j \in \mathbb{N}$ with $j \geq k-1$, which implies that $V_{j}$ is $\mathcal{L}_{\varphi \circ \pi_{1}}$-invariant by Lemma 3.13. As shown in the proof of Corollary 3.17 we may assume without loss of generality that $\mathcal{L}_{\varphi} \mathbb{1}=\mathbb{1}$ and thus $\mathcal{P}(\varphi)=0$.

The first inequality in (3.16) follows from Corollary 3.7 applied to $V=V_{j}$. The second inequality in (3.16) is an immediate consequence of the definition of the spectrum. The amenability dichotomy follows from Proposition 3.20 The equality $\log \rho\left(\mathcal{L}_{\varphi \circ \pi_{1}}\right)=\mathcal{P}(\varphi)$ follows from Lemma 3.5 (3).

In order to complete the proof, we now address (3.17) under the assumption that $\varphi$ is asymptotically symmetric with respect to $\Psi$. By Corollary 3.17, we then have that

$$
\sup _{g \in G}\left\{\mathcal{P}\left(\varphi, \Psi^{-1}\{g\} \cap \Sigma^{*}\right)\right\}=\log \rho\left(\left.\mathcal{L}_{\varphi \circ \pi_{1}}\right|_{V_{j}}\right) .
$$

Using that $\Psi\left(\Sigma^{*}\right)=G$ and item (3) of our standing assumptions, one easily verifies that the pressure $\mathcal{P}\left(\varphi, \Psi^{-1}\{g\} \cap \Sigma^{*}\right)$ is independent of $g \in G$, which completes the proof.

Corollary 3.22. Let $\varphi: \Sigma \rightarrow \mathbb{R}$ be $\mathcal{C}(k)$-measurable for some $k \in \mathbb{N}_{0}$ and let $\varphi$ be asymptotically symmetric with respect to $\Psi$. If $G$ is amenable, then $\mathcal{P}\left(\varphi, \Psi^{-1}\{\right.$ id $\left.\} \cap \Sigma^{*}\right)=\mathcal{P}(\varphi)$.

Proof. Using item (3) of our standing assumptions and that $\varphi$ is asymptotically symmetric with respect to $\Psi$, one verifies that $G^{\prime}:=\Psi\left(\Sigma^{*}\right)$ is a subgroup of $G$. Since $G$ is amenable, it is well known that $G^{\prime}$ is also amenable (see e.g. Woe00, Theorem $12.2(\mathrm{c})])$, and the corollary follows from Theorem 3.21 .

Remark 3.23. It is not difficult to extend Corollary 3.22 to arbitrary Hölder continuous potentials by approximating a Hölder continuous potential by a $\mathcal{C}(k)$ measurable potential and then letting $k$ tend to infinity. One obtains that, for 
an amenable group $G$ and for an asymptotically symmetric Hölder continuous potential $\varphi$, we have $\mathcal{P}\left(\varphi, \Psi^{-1}\{\operatorname{id}\} \cap \Sigma^{*}\right)=\mathcal{P}(\varphi)$. This was proved by the author in [Jae11, Theorem 5.3.11] and independently by Stadlbauer [Sta13, Theorem 4.1] in a slightly different setting. The converse implication of Corollary 3.22 for Hölder continuous potentials was proved recently in Sta13, Theorem 5.4] by extending ideas of Day ([Day64). A generalization of (3.17) in Theorem 3.21 for arbitrary Hölder continuous potentials still seems to be open.

\section{Proof of the Main Results}

For a linear GDMS $\Phi$ associated to $\mathbb{F}_{d}=\left\langle g_{1}, \ldots, g_{d}\right\rangle, d \geq 2$, we consider the Markov shift $\Sigma$ with alphabet $I:=\left\{g_{1}, g_{1}^{-1}, \ldots, g_{d}, g_{d}^{-1}\right\}$, given by

$$
\Sigma:=\left\{\omega \in I^{\mathbb{N}}: \omega_{i} \neq\left(\omega_{i+1}\right)^{-1} \text { for all } i \in \mathbb{N}\right\} .
$$

The involution $\kappa: \Sigma^{*} \rightarrow \Sigma^{*}$ is given by $\kappa(\omega):=\left(\omega_{n}^{-1}, \omega_{n-1}^{-1}, \ldots, \omega_{1}^{-1}\right)$, for all $n \in \mathbb{N}$ and $\omega \in \Sigma^{n}$.

For a normal subgroup $N$ of $\mathbb{F}_{d}$, we let $\Psi_{N}: I^{*} \rightarrow \mathbb{F}_{d} / N$ denote the unique semigroup homomorphism such that $\Psi_{N}(g)=g \bmod N$ for all $g \in I$. Clearly, we have that

$$
\Psi_{N}\left(\Sigma^{*}\right)=\mathbb{F}_{d} / N
$$

Since the assertions in Theorem 1.1 and Proposition 1.3 are clearly satisfied in the case that $N=\{\operatorname{id}\}$, we will from now on assume that $N \neq\{\operatorname{id}\}$. Using that $N$ is a normal subgroup of $\mathbb{F}_{d}$ and $d \geq 2$, one easily verifies that there exists a finite set $F \subset \Sigma^{*} \cap \Psi_{N}^{-1}\{\mathrm{id}\}$ with the following property:

For all $i, j \in I$ there exists $\tau \in F \cup\{\varnothing\}$ such that $i \tau j \in \Sigma^{*}$.

Note (4.2) implies that the group-extended Markov system $\left(\Sigma \times\left(\mathbb{F}_{d} / N\right), \sigma \rtimes \Psi_{N}\right)$ satisfies item (3) of our standing assumptions at the beginning of Section 4 . Hence, the results of Section 3 are applicable to the $\mathcal{C}(1)$-measurable potential $\varphi: \Sigma \rightarrow \mathbb{R}$, given by $\varphi_{\mid[g]}=\log \left(c_{\Phi}(g)\right)$ for all $g \in I$.

Proof of Theorem 1.1. Our aim is to apply Theorem 3.21 to the group-extended Markov system $\left(\Sigma \times\left(\mathbb{F}_{d} / N\right), \sigma \rtimes \Psi_{N}\right)$ and the $\mathcal{C}(1)$-measurable potential $s \varphi$ : $\Sigma \rightarrow \mathbb{R}$, for each $s \in \mathbb{R}$. By (4.1) and (4.2), we are left to show that $s \varphi$ is asymptotically symmetric with respect to $\Psi_{N}$. Since $\Phi$ is symmetric we have that $c_{\Phi}(\omega)=c_{\Phi}(\kappa(\omega))$, for all $\omega \in \Sigma^{*}$. Hence, for all $s \in \mathbb{R}, n \in \mathbb{N}$ and $g \in \mathbb{F}_{d} / N$, we have that

$$
\begin{aligned}
\sum_{\omega \in \Sigma^{n}: \Psi_{N}(\omega)=g} \exp \left(s S_{\omega} \varphi\right) & =\sum_{\omega \in \Sigma^{n}: \Psi_{N}(\omega)=g}\left(c_{\Phi}(\omega)\right)^{s}=\sum_{\omega \in \Sigma^{n}: \Psi_{N}(\omega)=g}\left(c_{\Phi}(\kappa(\omega))\right)^{s} \\
& =\sum_{\omega \in \Sigma^{n}: \Psi_{N}(\omega)=g^{-1}}\left(c_{\Phi}(\omega)\right)^{s}=\sum_{\omega \in \Sigma^{n}: \Psi_{N}(\omega)=g^{-1}} \exp \left(s S_{\omega} \varphi\right),
\end{aligned}
$$

which proves that $s \varphi$ is asymptotically symmetric with respect to $\Psi_{N}$. We are now in the position to apply Theorem 3.21 , which gives that amenability of $\mathbb{F}_{d} / N$ is equivalent to

$$
\mathcal{P}\left(s \varphi, \Psi_{N}^{-1}\{\operatorname{id}\} \cap \Sigma^{*}\right)=\mathcal{P}(s \varphi) .
$$


Since $\delta(N, \Phi)$ is equal to the unique zero of $s \mapsto \mathcal{P}\left(s \varphi, \Psi_{N}^{-1}\{\mathrm{id}\} \cap \Sigma^{*}\right)$ and $\delta\left(\mathbb{F}_{d}, \Phi\right)$ is equal to the unique zero of $s \mapsto \mathcal{P}(s \varphi)$ by Fact 2.3. we conclude that

$$
\delta\left(\mathbb{F}_{d}, \Phi\right)=\delta(N, \Phi) \text { if and only if } \mathbb{F}_{d} / N \text { is amenable. }
$$

The proof is complete.

For the proof of Theorem 1.2 we need the following lemma.

Lemma 4.1. Let $\Phi$ be a symmetric linear GDMS associated to $\mathbb{F}_{d}$. For every non-trivial normal subgroup $N$ of $\mathbb{F}_{d}$, we have that

$$
\sum_{h \in N}\left(c_{\Phi}(h)\right)^{\delta\left(\mathbb{F}_{d}, \Phi\right) / 2}=\infty .
$$

In particular, we have that $\delta(N, \Phi) \geq \delta\left(\mathbb{F}_{d}, \Phi\right) / 2$.

Proof. First observe that $N$ and $\Psi_{N}^{-1}\{\operatorname{id}\} \cap \Sigma^{*}$ are in one-to-one correspondence, which implies that

$$
\sum_{h \in N}\left(c_{\Phi}(h)\right)^{\delta\left(\mathbb{F}_{d}, \Phi\right) / 2}=\sum_{\omega \in \Psi_{N}^{-1}\{\operatorname{id}\} \cap \Sigma^{*}} \exp \left(\left(\delta\left(\mathbb{F}_{d}, \Phi\right) / 2\right) S_{\omega} \varphi\right) .
$$

For each $\omega \in \Sigma^{*}$, we can choose $\tau(\omega) \in F$ such that $\omega \tau(\omega) \kappa(\omega) \in \Sigma^{*}$ by making use of property (4.2). Further, we define the map $\Theta: \Sigma^{*} \rightarrow \Psi_{N}^{-1}\{$ id $\} \cap$ $\Sigma^{*}, \Theta(\omega):=\omega \tau(\omega) \kappa(\omega)$, which is at most card $(F)$-to-one. Moreover, setting $C:=\min \left\{S_{\tau} \varphi / 2: \tau \in F\right\}>-\infty$ and using that $\Phi$ is symmetric, we observe that $S_{\omega} \varphi+C=S_{\omega} \varphi / 2+S_{\kappa(\omega)} \varphi / 2+C \leq S_{\Theta(\omega)} \varphi / 2$, for each $\omega \in \Sigma^{*}$. Consequently, we have that

$$
\begin{aligned}
& \sum_{\omega \in \Psi_{N}^{-1}\{\operatorname{id}\} \cap \Sigma^{*}} \exp \left(\left(\delta\left(\mathbb{F}_{d}, \Phi\right) / 2\right) S_{\omega} \varphi\right) \\
\geq & \operatorname{card}(F)^{-1} \sum_{\omega \in \Sigma^{*}} \exp \left(\left(\delta\left(\mathbb{F}_{d}, \Phi\right) / 2\right) S_{\Theta(\omega)} \varphi\right) \\
\geq & \operatorname{card}(F)^{-1} \exp \left(\delta\left(\mathbb{F}_{d}, \Phi\right) C\right) \sum_{\omega \in \Sigma^{*}} \exp \left(\delta\left(\mathbb{F}_{d}, \Phi\right) S_{\omega} \varphi\right) .
\end{aligned}
$$

Finally, the existence of the Gibbs measure $\mu=\mu_{\delta\left(\mathbb{F}_{d}, \Phi\right) \varphi}$ implies that there exists a constant $C_{\mu}>0$ such that

$$
\sum_{\omega \in \Sigma^{*}} \exp \left(\delta\left(\mathbb{F}_{d}, \Phi\right) S_{\omega} \varphi\right) \geq C_{\mu} \sum_{\omega \in \Sigma^{*}} \mu([\omega])=C_{\mu} \sum_{n \in \mathbb{N}} \sum_{\omega \in \Sigma^{n}} \mu([\omega])=C_{\mu} \sum_{n \in \mathbb{N}} 1=\infty .
$$

Combining the latter estimate with (4.3), the proof is complete.

Proof of Theorem 1.2. By Theorem 1.1, the assertion is clearly true if $\mathbb{F}_{d} / N$ is amenable. We address the remaining case that $\mathbb{F}_{d} / N$ is non-amenable. Suppose for a contradiction that the claim is wrong. By Lemma 4.1, we obtain that

$$
\delta(N, \Phi)=\delta\left(\mathbb{F}_{d}\right) / 2 .
$$

For notational convenience, we set $G:=\mathbb{F}_{d} / N$ throughout this proof.

Consider the non-negative matrix $P \in \mathbb{R}^{(I \times G) \times(I \times G)}$, given by

$$
p\left(\left(v_{1}, g_{1}\right),\left(v_{2}, g_{2}\right)\right)= \begin{cases}c_{\Phi}\left(v_{1}\right)^{\delta(N, \Phi)}, & \text { if } v_{1} \neq v_{2}^{-1} \text { and } g_{2}=g_{1} \Psi_{N}\left(v_{1}\right) \\ 0 & \text { else. }\end{cases}
$$


By the assertions in (4.1) and (4.2), we have that $P$ is irreducible in the sense that for all $x, y \in I \times G$ there exists $n \in \mathbb{N}$ such that $p^{(n)}(x, y)>0$. Using the irreducibility of $P$ and that card $(I)=2 d<\infty$, we deduce from (4.4) and Lemma 4.1 that $P$ is $R$-recurrent with $R=1$ in the sense of Vere-Jones ([VJ62]; see also Seneta [Sen06, Definition 6.4]). That is, $P$ satisfies the following properties:

$$
\limsup _{n \rightarrow \infty}\left(p^{(n)}(x, y)\right)^{1 / n}=1 \text { and } \sum_{n \in \mathbb{N}} p^{(n)}(x, y)=\infty, \text { for all } x, y \in I \times G \text {. }
$$

Thus, by Sen06, Theorem 6.2], it follows that there exists a positive row vector $h \in \mathbb{R}^{I \times G}$ such that

$$
h P=h .
$$

It also follows from Sen06, Theorem 6.2] that the vector $h$ in (4.6) is unique up to a constant multiple. Next, we define the non-negative matrix $P_{h} \in \mathbb{R}^{(I \times G) \times(I \times G)}$, which is for all $x, y \in I \times G$ given by

$$
p_{h}(x, y)=p(y, x) h(y) / h(x) .
$$

It follows from (4.6) that $P_{h}$ is a transition matrix on $I \times G$. Further, we deduce from (4.5) that $P_{h}$ is 1-recurrent.

In order to derive a contradiction, we consider $P_{h}$ as a random walk on the graph $X_{1}$ associated to the group-extended Markov system $\left(\Sigma \times G, \sigma \rtimes \Psi_{N}\right)$ (see Definition 3.18) and we investigate the automorphisms of $X_{1}$. Let Aut $\left(X_{1}\right)$ denote the group of self-isometries of $\left(X_{1}, d_{X_{1}}\right)$, where $d_{X_{1}}$ denotes the graph metric on $X_{1}$. Note that each element $g \in G$ gives rise to an automorphism $\gamma_{g} \in \operatorname{Aut}\left(X_{1}\right)$, which is given by $\gamma_{g}(i, \tau):=(i, g \tau)$, for each $(i, \tau) \in I \times G$. The next step is to verify that also $\gamma_{g} \in \operatorname{Aut}\left(X_{1}, P_{h}\right)$, where we have set

$$
\operatorname{Aut}\left(X_{1}, P_{h}\right):=\left\{\gamma \in \operatorname{Aut}\left(X_{1}\right): P_{h}(x, y)=P_{h}(\gamma x, \gamma y) \text {, for all } x, y \in I \times G\right\} \text {. }
$$

Since $P$ has the property that $p(x, y)=p\left(\gamma_{g}(x), \gamma_{g}(y)\right)$, for all $x, y \in I \times G$ and $g \in$ $G$, it follows that the vector $h_{g} \in \mathbb{R}^{I \times G}$, given by $h_{g}(i, \tau):=h(i, g \tau),(i, \tau) \in I \times G$, satisfies $h_{g} P=h_{g}$ as well. Since the function $h$ in (4.6) is unique up to a constant multiple, we conclude that there exists a homomorphism $r: G \rightarrow \mathbb{R}^{+}$such that $h_{g}=r(g) h$, for each $g \in G$. Consequently, we have $p_{h}(x, y)=p_{h}\left(\gamma_{g}(x), \gamma_{g}(y)\right)$ for all $x, y \in I \times G$ and $g \in G$. Hence, $\gamma_{g} \in \operatorname{Aut}\left(X_{1}, P_{h}\right)$ for each $g \in G$. Since $\operatorname{card}(I)<\infty$, we deduce that $\left.\operatorname{Aut}\left(X_{1}, P_{h}\right)\right)$ acts with finitely many orbits on $X_{1}$.

In the terminology of Woe00 this is to say that $\left(X_{1}, P_{h}\right)$ is a quasi-transitive recurrent random walk. By [Woe00, Theorem 5.13] we then have that $X_{1}$ is a generalized lattice of dimension one or two. In particular, we have that $X_{1}$ has polynomial growth with degree one or two ([Woe00, Proposition 3.9]). Since $X_{1}$ is roughly isometric to the Cayley graph of $G$ by Lemma 3.19, we conclude that also $G$ has polynomial growth (see e.g. Woe00, Lemma 3.13]). This contradicts the well-known fact that each non-amenable group has exponential growth. The proof is complete.

Remark. The construction of the matrix $P_{h}$ and the verification of its invariance properties is analogous to the discussion of the $h$-process in Woe00, Proof of Theorem 7.8] and goes back to the work of Guivarc'h (Gui80, page 85]) on random walks on groups. However, note that in our case $P$ is in general not stochastic. 
Proof of Proposition 1.3. In order to investigate the radial limit sets of $N$, we introduce an induced GDMS $\tilde{\Phi}$ whose edge set consists of first return loops in the Cayley graph of $\mathbb{F}_{d} / N$. We define $\tilde{\Phi}:=\left(V,\left(X_{v}\right)_{v \in V}, \tilde{E}, \tilde{i}, \tilde{t},\left(\tilde{\phi}_{\omega}\right)_{\omega \in \tilde{E}}, \tilde{A}\right)$ as follows. The edge set $\tilde{E}$ and $\tilde{i}, \tilde{t}: \tilde{E} \rightarrow V$ are given by

$$
\begin{gathered}
\tilde{E}:=\left\{\omega=\left(v_{i}, w_{i}\right) \in \Sigma_{\Phi}^{*}: v_{1} \ldots v_{|\omega|} \in N, v_{1} \ldots v_{k} \notin N \text { for all } 1 \leq k<|\omega|\right\}, \\
\tilde{i}(\omega):=i\left(\omega_{1}\right), \tilde{t}(\omega):=t\left(\omega_{|\omega|}\right), \omega \in \tilde{E},
\end{gathered}
$$

the matrix $\tilde{A}=\left(\tilde{a}\left(\omega, \omega^{\prime}\right)\right) \in\{0,1\}^{\tilde{E} \times \tilde{E}}$ satisfies $\tilde{a}\left(\omega, \omega^{\prime}\right)=1$ if and only if $a\left(\omega_{|\omega|}, \omega_{1}^{\prime}\right)=1$, and the family $\left(\tilde{\phi}_{\omega}\right)_{\omega \in \tilde{E}}$ is given by $\tilde{\phi}_{\omega}:=\phi_{\omega}, \omega \in \tilde{E}$. One immediately verifies that $\tilde{\Phi}$ is a conformal GDMS. Note that there are canonical embeddings from $\Sigma_{\tilde{\Phi}}$ into $\Sigma_{\Phi}$ and from $\Sigma_{\tilde{\Phi}}^{*}$ into $\Sigma_{\Phi}^{*}$, which we will both indicate by omitting the tilde, that is, $\tilde{\omega} \mapsto \omega$. For the coding maps $\pi_{\tilde{\Phi}}: \Sigma_{\tilde{\Phi}} \rightarrow J(\tilde{\Phi})$ and $\pi_{\Phi}: \Sigma_{\Phi} \rightarrow J(\Phi)$ we have $\pi_{\tilde{\Phi}}(\tilde{\omega})=\pi_{\Phi}(\omega)$, for each $\tilde{\omega} \in \Sigma_{\tilde{\Phi}}$. The following relations between the limit set of $\tilde{\Phi}$ and the radial limit sets of $N$ are straightforward to prove. We have that

$$
J^{*}(\tilde{\Phi}) \subset \Lambda_{\mathrm{ur}}(N, \Phi) \subset \Lambda_{\mathrm{r}}(N, \Phi) \subset J(\tilde{\Phi}) \cup \bigcup_{\eta \in \Sigma_{\Phi}^{*}, \tilde{\omega} \in \Sigma_{\tilde{\Phi}}: \eta \omega \in \Sigma_{\Phi}} \phi_{\eta}\left(\pi_{\tilde{\Phi}}(\tilde{\omega})\right) .
$$

Note that the right-hand side in the latter chain of inclusions can be written as a countable union of images of $J(\tilde{\Phi})$ under Lipschitz continuous maps. Since Lipschitz continuous maps do not increase Hausdorff dimension and since Hausdorff dimension is stable under countable unions, we obtain

$$
\text { (4.7) } \operatorname{dim}_{H}\left(J^{*}(\tilde{\Phi})\right) \leq \operatorname{dim}_{H}\left(\Lambda_{\mathrm{ur}}(N, \Phi)\right) \leq \operatorname{dim}_{H}\left(\Lambda_{\mathrm{r}}(N, \Phi)\right) \leq \operatorname{dim}_{H}(J(\tilde{\Phi})) .
$$

Since the incidence matrix of $\tilde{\Phi}$ is finitely irreducible by property (4.2), the generalised Bowen's formula (Theorem 2.9) implies $\operatorname{dim}_{H}\left(J^{*}(\tilde{\Phi})\right)=\operatorname{dim}_{H}(J(\tilde{\Phi}))$, so equality holds in (4.7).

The final step is to show that $\operatorname{dim}_{H}(J(\tilde{\Phi}))=\delta(N, \Phi)$. By Theorem 2.9 and Fact 2.3, we have

$$
\operatorname{dim}_{H}(J(\tilde{\Phi}))=\mathcal{P}_{-\tilde{\zeta_{\Phi}}}\left(0, \Sigma_{\tilde{\Phi}}^{*}\right)=\inf \left\{s \in \mathbb{R}: \sum_{\tilde{\omega} \in \Sigma_{\tilde{\Phi}}^{*}} \mathrm{e}^{s S_{\tilde{\omega}} \zeta_{\tilde{\Phi}}}<\infty\right\} .
$$

Since the elements $\tilde{\omega} \in \Sigma_{\tilde{\Phi}}^{*}$ are in one-to-one correspondence with $\omega \in \mathcal{C}_{N}$, where $\mathcal{C}_{N}$ is given by

$$
\mathcal{C}_{N}:=\left\{\omega=\left(v_{i}, w_{i}\right) \in \Sigma_{\Phi}^{*}: v_{1} \ldots v_{|\omega|} \in N\right\},
$$

and using that $S_{\tilde{\omega}} \zeta_{\tilde{\Phi}}=S_{\omega} \zeta_{\Phi}$ for all $\tilde{\omega} \in \Sigma_{\tilde{\Phi}}^{*}$, we conclude that

$$
\operatorname{dim}_{H}(J(\tilde{\Phi}))=\inf \left\{s \in \mathbb{R}: \sum_{\omega \in \mathcal{C}_{N}} \mathrm{e}^{s S_{\omega} \zeta_{\Phi}}<\infty\right\} .
$$

Finally, since the map from $\mathcal{C}_{N}$ onto $N$, given by $\omega=\left(\left(v_{1}, w_{1}\right),\left(v_{2}, w_{2}\right), \ldots,\left(v_{n}, w_{n}\right)\right)$ $\mapsto v_{1} v_{2} \cdots v_{n}$ for $n \in \mathbb{N}$, is $(2 d-1)$-to-one, and since $S_{\omega} \zeta_{\Phi}=c_{\Phi}\left(v_{1} \ldots v_{n}\right)$ for all 
$\omega \in \mathcal{C}_{N}$, it follows that

$$
\operatorname{dim}_{H}(J(\tilde{\Phi}))=\inf \left\{s \in \mathbb{R}: \sum_{g \in N}\left(c_{\Phi}(g)\right)^{s}<\infty\right\}=\delta(N, \Phi),
$$

which completes the proof.

\section{Kleinian GROUPS}

In this section we give a more detailed discussion of Kleinian groups and how these relate to the concept of a GDMS. In particular, in Proposition [5.6 we will give the motivation for our definition of the radial limit set in the context of a GDMS associated to the free group (see Definition 2.10).

In the following we let $G \subset \operatorname{Con}(m)$ denote a non-elementary, torsion-free Kleinian group acting properly discontinuously on the $(m+1)$-dimensional hyperbolic space $\mathbb{D}^{m+1}$, where Con $(m)$ denotes the set of orientation preserving conformal automorphisms of $\mathbb{D}^{m+1}$. The limit set $L(G)$ of $G$ is the set of accumulation points with respect to the Euclidean topology on $\mathbb{R}^{m+1}$ of the $G$-orbit of some arbitrary point in $\mathbb{D}^{m+1}$, that is, for each $z \in \mathbb{D}^{m+1}$ we have

$$
L(G)=\overline{G(z)} \backslash G(z),
$$

where the closure is taken with respect to the Euclidean topology on $\mathbb{R}^{m+1}$. Clearly, $L(G)$ is a subset of $\mathbb{S}$. For more details on Kleinian groups and their limit sets, we refer to [Bea95, Mas88, Nic89, MT98, Str06].

Let us recall the definition of the following important subsets of $L(G)$, namely the radial and the uniformly radial limit set of $G$. Here, $s_{\xi} \subset \mathbb{D}^{m+1}$ denotes the hyperbolic ray from 0 to $\xi$ and $B(x, r):=\left\{z \in \mathbb{D}^{m+1}: d(z, x)<r\right\} \subset \mathbb{D}^{m+1}$ denotes the open hyperbolic ball of radius $r$ centred at $x$, where $d$ denotes the hyperbolic metric on $\mathbb{D}^{m+1}$.

Definition 5.1. For a Kleinian group $G$ the radial and the uniformly radial limit set of $G$ are given by

$$
L_{\mathrm{r}}(G):=\left\{\xi \in L(G): \exists c>0 \text { such that } s_{\xi} \cap B(g(0), c) \neq \varnothing\right.
$$

$$
\text { for infinitely many } g \in G\} \text {, }
$$

and

$$
L_{\mathrm{ur}}(G):=\left\{\xi \in L(G): \exists c>0 \text { such that } s_{\xi} \subset \bigcup_{g \in G} B(g(0), c)\right\} .
$$

A Kleinian group $G$ is said to be geometrically finite if the action of $G$ on $\mathbb{D}^{m+1}$ admits a fundamental polyhedron with finitely many sides. We denote by $E_{G}$ the set of points in $\mathbb{D}^{m+1}$ which lie on a geodesic connecting any two limit points in $L(G)$. The convex hull of $E_{G}$, which we will denote by $C_{G}$, is the minimal hyperbolic convex subset of $\mathbb{D}^{m+1}$ containing $E_{G}$. $G$ is called convex cocompact ([Nic89, page 7]) if the action of $G$ on $C_{G}$ has a compact fundamental domain in $\mathbb{D}^{m+1}$.

The following class of Kleinian groups gives the main motivation for our definition of a GDMS associated to the free group (see also [Mas88, X.H]). 
Definition 5.2. Let $d \geq 2$ and let $\mathcal{D}:=\left\{\left(D_{n}^{j}\right): n \in\{1, \ldots, d\}, j \in\{-1,1\}\right\}$ be a family of pairwise disjoint compact Euclidean balls $D_{n}^{j} \subset \mathbb{R}^{m+1}$ which intersect $\mathbb{S}^{m}$ orthogonally such that $\operatorname{diam}\left(D_{n}\right)=\operatorname{diam}\left(D_{n}^{-1}\right)$. For each $n \in\{1, \ldots, d\}$, let $g_{n} \in \operatorname{Con}(m)$ be the unique hyperbolic element such that $g_{n}\left(\mathbb{D}^{m+1} \cap \partial D_{n}^{-1}\right)=$ $\mathbb{D}^{m+1} \cap \partial D_{n}$, where $\partial D_{n}^{j}$ denotes the boundary of $D_{n}^{j}$ with respect to the Euclidean metric on $\mathbb{R}^{m+1}$. Then $G:=\left\langle g_{1}, \ldots, g_{d}\right\rangle$ is referred to as the Kleinian group of Schottky type generated by $\mathcal{D}$.

Note that a Kleinian group of Schottky type $G=\left\langle g_{1}, \ldots, g_{d}\right\rangle$ is algebraically a free group. The following construction of a particular GDMS associated to the free group $\left\langle g_{1}, \ldots, g_{d}\right\rangle$ is canonical.

Definition 5.3. Let $G=\left\langle g_{1}, \ldots, g_{d}\right\rangle$ be a Kleinian group of Schottky type generated by $\mathcal{D}$. The canonical GDMS $\Phi_{G}$ associated to $G$ is the GDMS associated to the free group $\left\langle g_{1}, \ldots, g_{d}\right\rangle$ which satisfies $X_{g_{n}^{j}}:=\left(\mathbb{D}^{m+1} \cup \mathbb{S}^{m}\right) \cap D_{n}^{j}$, for each $n \in\{1, \ldots, d\}$ and $j \in\{-1,1\}$, and for which the contractions $\phi_{(v, w)}: X_{w} \rightarrow X_{v}$ are given by $\phi_{(v, w)}:=\left.v\right|_{X_{w}}$, for each $(v, w) \in E$.

For the following fact we refer to [MU03, Theorem 5.1.6].

Fact 5.4. For a Kleinian group of Schottky type $G$ we have that $L(G)=J\left(\Phi_{G}\right)$.

Remark 5.5. We remark that without our assumption on $G$ that $\operatorname{diam}\left(D_{n}\right)=$ $\operatorname{diam}\left(D_{n}^{-1}\right)$, for each $n \in\{1, \ldots, d\}$ in Definition [5.2, the generators of the associated GDMS $\Phi_{G}$ may fail to be contractions. However, in that case, by taking sufficiently high iterates of the generators, we can pass to a finite index subgroup of $G$, for which there exists a set $\mathcal{D}$ as in Definition 5.2

The following brief discussion of the geometry of a Kleinian group of Schottky type $G$ contains nothing that is not well known; however, the reader might like to recall a few of its details. Let $\Phi_{G}$ denote the canonical GDMS associated to $G$. Recall that for the half-spaces

$$
H_{v}:=\left\{z \in \mathbb{D}^{m+1}: d(z, 0)<d(z, v(0))\right\}, \text { for each } v \in V,
$$

we have that the set

$$
F:=\bigcap_{v \in V} H_{v}
$$

is referred to as a Dirichlet fundamental domain for $G$. That $F$ is a fundamental domain for $G$ means that $F$ is an open set which satisfies the conditions

$$
\bigcup_{g \in G} g\left(\bar{F} \cap \mathbb{D}^{m+1}\right)=\mathbb{D}^{m+1} \text { and } g(F) \cap h(F)=\varnothing \text { for all } g, h \in G \text { with } g \neq h \text {. }
$$

For $\omega=\left(v_{k}, w_{k}\right)_{k \in \mathbb{N}} \in \Sigma_{\Phi_{G}}$ and $\pi_{\Phi_{G}}(\omega)=\xi$, we have that the ray $s_{\xi}$ successively passes through the fundamental domains $F, v_{1}(F), v_{1} v_{2}(F), \ldots$

We also make use of the fact that a Kleinian group of Schottky type $G$ is convex cocompact. This follows from a theorem due to Beardon and Maskit (BM74, [Str06, Theorem 2]), since $G$ is geometrically finite and $L(G)$ contains no parabolic fixed points (cf. [Rat06, Theorem 12.27]). Clearly, if $G$ is convex cocompact, then there exists $R_{G}>0$ such that

$$
C_{G} \cap g \bar{F} \subset B\left(g(0), R_{G}\right), \text { for all } g \in G .
$$

In particular, we have that $L_{\mathrm{ur}}(G)=L_{\mathrm{r}}(G)=L(G)$. 
Using the fact that $G$ acts properly discontinuously on $\mathbb{D}^{m+1}$ and that $G$ is convex cocompact, one easily verifies that for each $r>0$ there exists a finite set $\Gamma \subset G$ such that

$$
B(0, r) \cap C_{G} \subset \bigcup_{\gamma \in \Gamma} \gamma \bar{F}
$$

The next proposition provides the main motivation for our definition of the (uniformly) radial limit set of a normal subgroup $N$ of $\mathbb{F}_{d}$ with respect to a GDMS associated to $\mathbb{F}_{d}$.

Proposition 5.6. Let $G$ be a Kleinian group of Schottky type and let $\Phi_{G}$ denote the canonical GDMS associated to $G$. For every non-trivial normal subgroup $N$ of $G$, we have that

$$
L_{\mathrm{r}}(N)=\Lambda_{\mathrm{r}}\left(N, \Phi_{G}\right) \text { and } L_{\mathrm{ur}}(N)=\Lambda_{\mathrm{ur}}\left(N, \Phi_{G}\right) .
$$

Proof. Let us begin by proving that $\Lambda_{\text {ur }}\left(N, \Phi_{G}\right) \subset L_{\text {ur }}(N)$. To start, let $\xi \in$ $\Lambda_{\mathrm{ur}}\left(N, \Phi_{G}\right)$ be given. By the definition of $\Lambda_{\mathrm{ur}}\left(N, \Phi_{G}\right)$, there exists $\omega=\left(v_{k}, w_{k}\right)_{k \in \mathbb{N}}$ $\in \Sigma_{\Phi_{G}}$ and a finite set $\Gamma \subset G$ such that $\pi_{\Phi_{G}}(\omega)=\xi$ and $v_{1} v_{2} \cdots v_{k} \in N \Gamma$, for all $k \in \mathbb{N}$. Hence, using (5.1), it follows that

$$
s_{\xi} \subset \bigcup_{h \in N} \bigcup_{\gamma \in \Gamma} B\left(h \gamma(0), R_{G}\right) .
$$

Note that for each $h \in N, \gamma \in \Gamma$ and $x \in B\left(h \gamma(0), R_{G}\right)$ we have

$$
d(h(0), x) \leq d(h(0), h \gamma(0))+d(h \gamma(0), x)<\max \{d(0, \gamma(0)): \gamma \in \Gamma\}+R_{G},
$$

which implies that

$$
\bigcup_{h \in N} \bigcup_{\gamma \in \Gamma} B\left(h \gamma(0), R_{G}\right) \subset \bigcup_{h \in N} B\left(h(0), R_{G}+\max \{d(0, \gamma(0)): \gamma \in \Gamma\}\right) .
$$

Thus, $\xi \in L_{\text {ur }}(N)$.

For the converse inclusion, let $\xi \in L_{\mathrm{ur}}(N)$ be given. Then, by the definition of $L_{\text {ur }}(N)$, there exists a constant $c:=c(\xi)>0$ such that

$$
s_{\xi} \subset \bigcup_{h \in N} B(h(0), c) .
$$

Hence, by (5.2), there exists a finite set $\Gamma \subset G$ such that $s_{\xi} \subset \bigcup_{h \in N} \bigcup_{\gamma \in \Gamma} h \gamma \bar{F}$. We conclude that for $\omega=\left(v_{k}, w_{k}\right)_{k \in \mathbb{N}} \in \Sigma_{\Phi_{G}}$ with $\pi_{\Phi_{G}}(\omega)=\xi$ we have that $\left\{v_{1} v_{2} \cdots v_{k}: k \in \mathbb{N}\right\} \subset N \Gamma$, and hence $\xi \in \Lambda_{\text {ur }}\left(N, \Phi_{G}\right)$.

Let us now address the inclusion $\Lambda_{\mathrm{r}}\left(N, \Phi_{G}\right) \subset L_{\mathrm{r}}(N)$. For this, let $\xi \in$ $\Lambda_{\mathrm{r}}\left(N, \Phi_{G}\right)$ be given. By the definition of $\Lambda_{\mathrm{r}}\left(N, \Phi_{G}\right)$, there exists $\omega=\left(v_{k}, w_{k}\right)_{k \in \mathbb{N}} \in$ $\Sigma_{\Phi_{G}}$, an element $\gamma \in G$, a sequence $\left(h_{k}\right)_{k \in \mathbb{N}}$ of pairwise distinct elements in $N$ and a sequence $\left(n_{k}\right)_{k \in \mathbb{N}}$ tending to infinity such that $\pi_{\Phi_{G}}(\omega)=\xi$ and $v_{1} v_{2} \cdots v_{n_{k}}=h_{k} \gamma$, for all $k \in \mathbb{N}$. Using (5.1) it follows that $s_{\xi} \cap B\left(h_{k} \gamma(0), R_{G}\right) \neq \varnothing$, for all $k \in \mathbb{N}$. Since $B\left(h_{k} \gamma(0), R_{G}\right) \subset B\left(h_{k}(0), R_{G}+d(0, \gamma(0))\right)$ for all $k \in \mathbb{N}$, we obtain that also $s_{\xi} \cap B\left(h_{k}(0), R_{G}+d(0, \gamma(0))\right) \neq \varnothing$. We have thus shown that $\xi \in L_{\mathrm{r}}(N)$.

Finally, let us demonstrate that $L_{\mathrm{r}}(N) \subset \Lambda_{\mathrm{r}}\left(N, \Phi_{G}\right)$. To that end, pick an arbitrary $\xi \in L_{\mathrm{r}}(N)$ and let $\omega=\left(v_{k}, w_{k}\right)_{k \in \mathbb{N}} \in \Sigma_{\Phi_{G}}$ with $\pi_{\Phi_{G}}(\omega)=\xi$ be given. Then, by the definition of $L_{\mathrm{r}}(N)$, there exists $c>0$ and a sequence $\left(h_{k}\right)_{k \in \mathbb{N}}$ of pairwise distinct elements in $N$ such that $s_{\xi} \cap B\left(h_{k}(0), c\right) \neq \varnothing$, for all $k \in \mathbb{N}$. 
Using (5.2) we deduce that there exists a finite set $\Gamma \subset G$ such that for all $k \in \mathbb{N}$ we have

$$
s_{\xi} \cap B\left(h_{k}(0), c\right) \cap \bigcup_{\gamma \in \Gamma} h_{k} \gamma \bar{F} \neq \varnothing .
$$

Since $\Gamma$ is finite, there exist $\gamma_{0} \in \Gamma$ and sequences $\left(n_{k}\right)_{k \in \mathbb{N}}$ and $\left(l_{k}\right)_{k \in \mathbb{N}}$ tending to infinity such that $s_{\xi} \cap B\left(h_{n_{k}}(0), c\right) \cap h_{n_{k}} \gamma_{0} \bar{F} \neq \varnothing$ and $v_{1} v_{2} \cdots v_{l_{k}}=h_{n_{k}} \gamma_{0}$, for all $k \in \mathbb{N}$. Hence, $\xi \in \Lambda_{\mathrm{r}}\left(N, \Phi_{G}\right)$.

\section{ACKNOWLEDGEMENT}

Parts of this paper constitute certain parts of the author's doctoral thesis supervised by Marc Kesseböhmer at the University of Bremen. The author would like to express his deep gratitude to Marc Kesseböhmer and Bernd Stratmann for their support and many fruitful discussions. The author thanks an anonymous referee for the careful reading of the manuscript and for valuable comments on the exposition of this paper. Final thanks go to Sara Munday for helping to improve the presentation of the paper significantly.

\section{REFERENCES}

[AD00] Jon Aaronson and Manfred Denker, On exact group extensions, Sankhyā Ser. A 62 (2000), no. 3, 339-349. Ergodic theory and harmonic analysis (Mumbai, 1999). MR:1803461 (2001m:37011)

[AD02] Jon Aaronson and Manfred Denker, Group extensions of Gibbs-Markov maps, Probab. Theory Related Fields 123 (2002), no. 1, 38-40, DOI 10.1007/s004400100173. MR 1906436 (2003f:37010)

[Bea95] Alan F. Beardon, The geometry of discrete groups, Graduate Texts in Mathematics, vol. 91, Springer-Verlag, New York, 1995. Corrected reprint of the 1983 original. MR1393195 (97d:22011)

[Ber98] George M. Bergman, An invitation to general algebra and universal constructions, Henry Helson, Berkeley, CA, 1998. MR.1650275 (99h:18001)

[BJ97] Christopher J. Bishop and Peter W. Jones, Hausdorff dimension and Kleinian groups, Acta Math. 179 (1997), no. 1, 1-39, DOI 10.1007/BF02392718. MR.1484767 (98k:22043)

[BM74] Alan F. Beardon and Bernard Maskit, Limit points of Kleinian groups and finite sided fundamental polyhedra, Acta Math. 132 (1974), 1-12. MR0333164 (48 \#11489)

[Bow75] Rufus Bowen, Equilibrium states and the ergodic theory of Anosov diffeomorphisms, Lecture Notes in Mathematics, Vol. 470, Springer-Verlag, Berlin, 1975. MR0442989 (56 \#1364)

[Bro85] Robert Brooks, The bottom of the spectrum of a Riemannian covering, J. Reine Angew. Math. 357 (1985), 101-114, DOI 10.1515/crll.1985.357.101. MR783536 (86h:58138)

[BTMT12] Petra Bonfert-Taylor, Katsuhiko Matsuzaki, and Edward C. Taylor, Large and small covers of a hyperbolic manifold, J. Geom. Anal. 22 (2012), no. 2, 455-470, DOI 10.1007/s12220-010-9204-6. MR2891734

[Coh82] Joel M. Cohen, Cogrowth and amenability of discrete groups, J. Funct. Anal. 48 (1982), no. 3, 301-309, DOI 10.1016/0022-1236(82)90090-8. MR678175 (85e:43004)

[Day49] M. M. Day, Means on semigroups and groups, Bull. Amer. Math. Soc. 55 (1949), 1054-1055.

[Day64] Mahlon Marsh Day, Convolutions, means, and spectra, Illinois J. Math. 8 (1964), 100-111. MR0159230 (28 \#2447)

[DK86] J. Dodziuk and W. S. Kendall, Combinatorial Laplacians and isoperimetric inequality, From local times to global geometry, control and physics (Coventry, 1984/85), Pitman Res. Notes Math. Ser., vol. 150, Longman Sci. Tech., Harlow, 1986, pp. 68-74. MR:894523 (88h:58118) 
[Dod84] Jozef Dodziuk, Difference equations, isoperimetric inequality and transience of certain random walks, Trans. Amer. Math. Soc. 284 (1984), no. 2, 787-794, DOI 10.2307/1999107. MR743744 (85m:58185)

[Føl55] Erling Følner, On groups with full Banach mean value, Math. Scand. 3 (1955), 243254. MR0079220(18,51f)

[FS04] Kurt Falk and Bernd O. Stratmann, Remarks on Hausdorff dimensions for transient limit sets of Kleinian groups, Tohoku Math. J. (2) 56 (2004), no. 4, 571-582. MR2097162 (2005g:30053)

[Ger88] Peter Gerl, Random walks on graphs with a strong isoperimetric property, J. Theoret. Probab. 1 (1988), no. 2, 171-187, DOI 10.1007/BF01046933. MR938257 (89g:60216)

[Gui80] Y. Guivarc'h, Sur la loi des grands nombres et le rayon spectral d'une marche aléatoire (French, with English summary), Conference on Random Walks (Kleebach, 1979), Astérisque, vol. 74, Soc. Math. France, Paris, 1980, pp. 47-98, 3. MR588157 (82g:60016)

[Jae11] Johannes Jaerisch, Thermodynamic formalism for group-extended Markov systems with applications to Fuchsian groups, Doctoral dissertation at the University Bremen (2011).

[Jae13] Johannes Jaerisch, A lower bound for the exponent of convergence of normal subgroups of Kleinian groups, J. Geom. Anal., online first (2013).

[Jae14a] Johannes Jaerisch, Group-extended Markov systems, amenability, and the PerronFrobenius operator, Proc. Amer. Math. Soc. (2014) to appear.

[Jae14b] Johannes Jaerisch, Recurrence and pressure for group extensions, Ergodic Theory and Dynamical Systems (2014) to appear.

[Jae14c] Johannes Jaerisch, Conformal fractals for normal subgroups of free groups, Conform. Geom. Dyn. 18 (2014), 31-55. MR3175016

[JKL14] Johannes Jaerisch, Marc Kesseböhmer, and Sanaz Lamei, Induced topological pressure for countable state Markov shifts, Stoch. Dyn. 14 (2014), no. 2. MR3190211

[Kai92] Vadim A. Kaimanovich, Dirichlet norms, capacities and generalized isoperimetric inequalities for Markov operators, Potential Anal. 1 (1992), no. 1, 61-82, DOI 10.1007/BF00249786. MR1245225 (94i:31012)

[Kes59a] Harry Kesten, Full Banach mean values on countable groups, Math. Scand. 7 (1959), 146-156. MR0112053 (22 \#2911)

[Kes59b] Harry Kesten, Symmetric random walks on groups, Trans. Amer. Math. Soc. 92 (1959), 336-354. MR0109367 (22 \#253)

[Koo31] B. O. Koopman, Hamiltonian systems and transformation in Hilbert space, Proceedings of the National Academy of Sciences of the United States of America, vol. 17, 1931.

[LM94] Andrzej Lasota and Michael C. Mackey, Chaos, fractals, and noise, 2nd ed., Applied Mathematical Sciences, vol. 97, Springer-Verlag, New York, 1994. Stochastic aspects of dynamics. MR 1244104 (94j:58102)

[Mas88] Bernard Maskit, Kleinian groups, Grundlehren der Mathematischen Wissenschaften [Fundamental Principles of Mathematical Sciences], vol. 287, Springer-Verlag, Berlin, 1988. MR959135 (90a:30132)

[Moh88] Bojan Mohar, Isoperimetric inequalities, growth, and the spectrum of graphs, Linear Algebra Appl. 103 (1988), 119-131, DOI 10.1016/0024-3795(88)90224-8. MR943998 (89k:05071)

[MT98] Katsuhiko Matsuzaki and Masahiko Taniguchi, Hyperbolic manifolds and Kleinian groups, Oxford Mathematical Monographs, The Clarendon Press Oxford University Press, New York, 1998. Oxford Science Publications. MR.1638795 (99g:30055)

[MU03] R. D. Mauldin and M. Urbański, Graph directed Markov systems, Cambridge Tracts in Mathematics, vol. 148, Cambridge, 2003. MR2003772

[Neu29] J. v. Neumann, Zur allgemeinen Theorie des Masses, Fund. Math. 13 (1929), 73-116.

[Nic89] Peter J. Nicholls, The ergodic theory of discrete groups, London Mathematical Society Lecture Note Series, vol. 143, Cambridge University Press, Cambridge, 1989. MR.1041575 (91i:58104)

[OW07] Ronald Ortner and Wolfgang Woess, Non-backtracking random walks and cogrowth of graphs, Canad. J. Math. 59 (2007), no. 4, 828-844, DOI 10.4153/CJM-2007-035-1. MR2338235 (2008h:05057) 
[Pól21] G. Pólya, Über eine Aufgabe der Wahrscheinlichkeitsrechnung betreffend die Irrfahrt im Straßennetz, Math. Ann. 84 (1921), no. 1-2, 149-160. MR.1512028

[Rat06] John G. Ratcliffe, Foundations of hyperbolic manifolds, 2nd ed., Graduate Texts in Mathematics, vol. 149, Springer, New York, 2006. MR2249478(2007d:57029)

[Rob05] Thomas Roblin, Un théorème de Fatou pour les densités conformes avec applications aux revêtements galoisiens en courbure négative (French, with French summary), Israel J. Math. 147 (2005), 333-357, DOI 10.1007/BF02785371. MR2166367 (2006i:37065)

[RU08] Mario Roy and Mariusz Urbański, Real analyticity of Hausdorff dimension for higher dimensional hyperbolic graph directed Markov systems, Math. Z. 260 (2008), no. 1, 153-175, DOI 10.1007/s00209-007-0267-4. MR2413348(2009m:37064)

[Rud73] Walter Rudin, Functional analysis, McGraw-Hill Book Co., New York, 1973. McGrawHill Series in Higher Mathematics. MR0365062 (51 \#1315)

[Rue69] David Ruelle, Statistical mechanics: Rigorous results, W. A. Benjamin, Inc., New York-Amsterdam, 1969. MR0289084 (44 \#6279)

[Sen06] E. Seneta, Non-negative matrices and Markov chains, Springer Series in Statistics, Springer, New York, 2006. Revised reprint of the second (1981) edition [SpringerVerlag, New York; MR0719544]. MR2209438

[Sha07] Richard Sharp, Critical exponents for groups of isometries, Geom. Dedicata 125 (2007), 63-74, DOI 10.1007/s10711-007-9137-9. MR2322540 (2008f:20107)

[Sta13] Manuel Stadlbauer, An extension of Kesten's criterion for amenability to topological Markov chains, Adv. Math. 235 (2013), 450-468, DOI 10.1016/j.aim.2012.12.004. MR3010065

[Str04] Bernd O. Stratmann, The exponent of convergence of Kleinian groups; on a theorem of Bishop and Jones, Fractal geometry and stochastics III, Progr. Probab., vol. 57, Birkhäuser, Basel, 2004, pp. 93-107. MR2087134 (2005h:20114)

[Str06] Bernd O. Stratmann, Fractal geometry on hyperbolic manifolds, Non-Euclidean geometries, Math. Appl. (N. Y.), vol. 581, Springer, New York, 2006, pp. 227-247, DOI 10.1007/0-387-29555-0_12. MR2191250 (2006g:37038)

[VJ62] D. Vere-Jones, Geometric ergodicity in denumerable Markov chains, Quart. J. Math. Oxford Ser. (2) 13 (1962), 7-28. MR0141160 (25 \#4571)

[Wal82] Peter Walters, An introduction to ergodic theory, Graduate Texts in Mathematics, vol. 79, Springer-Verlag, New York, 1982. MR648108 (84e:28017)

[Woe00] Wolfgang Woess, Random walks on infinite graphs and groups, Cambridge Tracts in Mathematics, vol. 138, Cambridge University Press, Cambridge, 2000. MR,1743100 (2001k:60006)

Department of Mathematics, Graduate School of Science, Osaka University, 1-1 Machikaneyama, TOYONAKA, OSAKA, 560-0043 JAPAN

E-mail address: jaerisch@cr.math.sci.osaka-u.ac.jp 